\title{
Leveraging inter-tourists interactions via chatbots to bridge academia, tourism industries and future societies
}

\author{
Davide Calvaresi, Ahmed Ibrahim, Jean-Paul Calbimonte, Emmanuel Fragniere, \\ Roland Schegg and Michael Ignaz Schumacher
}

\begin{abstract}
Purpose - The tourism and hospitality sectors are experiencing radical innovation boosted by the advancements in Information and Communication Technologies. Increasingly sophisticated chatbots are introducing novel approaches, re-shaping the dynamics among tourists and service providers, and fostering a remarkable behavioral change in the overall sector. Therefore, the objective of this paper is two-folded: (1) to highlight the academic and industrial standing points with respect to the current chatbots designed/deployed in the tourism sector and (2) to develop a proof-of-concept embodying the most prominent opportunities in the tourism sector.

Design/methodology/approach - This work elaborates on the outcomes of a Systematic Literature Review (SLR) and a Focus Group (FG) composed of experts from the tourism industry. Moreover, it presents a proof-ofconcept relying on the outcomes obtained from both SLR and FG. Eventually, the proof-of-concept has been tested with experts and practitioners of the tourism sector.

Findings - Among the findings elicited by this paper, we can mention the quick evolution of chatbot-based solutions, the need for continuous investments, upskilling, system innovation to tackle the eTourism challenges and the shift toward new dimensions (i.e. tourist-to-tourist-to-chatbot and personalized multi-stakeholder systems). In particular, we focus on the need for chatbot-based activity and thematic aggregation for nextgeneration tourists and service providers.

Originality/value - Both academic- and industrial-centered findings have been structured and discussed to foster the practitioners' future research. Moreover, the proof-of-concept presented in the paper is the first of its kind, which raised considerable interest from both technical and business-planning perspectives.
\end{abstract}

Keywords Chatbots, Social chatbots, Virtual assistant, Tourism industry, Tourism academia, Industry focus group

Paper type Research paper

\section{Introduction}

Information and Communication Technologies (ICTS) have fundamentally transformed the dynamics and business models of the tourism and hospitality industry (Popesku and Zlatanov, 2019). In particular, user experience in tourism applications and systems has been re-shaped, extending the service providers' competition to new levels and forms. Traditionally, the customer's satisfaction and, eventually, loyalty to the service provider or brand strongly depended on the experiences' uniqueness. However, the impact of convenient and always available (i.e. 24/7) services and communication means is skyrocketing (Buhalis and Yen, 2020). These increased availability expectations have been translated into the development of different online services and mobile applications intending to facilitate and automate reservations and bookings while optimizing logistics. Overall, many of these mobile applications helped to boost the digitalization
Davide Calvaresi,

Ahmed Ibrahim,

Jean-Paul Calbimonte,

Emmanuel Fragniere,

Roland Schegg and

Michael Ignaz Schumacher

are all based at the University

of Applied Sciences and Arts

Western Switzerland

HES-SO, Sierre, Switzerland.
Received 5 January 2021
Revised 21 June 2021
Accepted 24 June 2021
C Davide Calvaresi, Ahmed
Ibrahim, Jean-Paul Calbimonte,
Emmanuel Fragniere, Roland
Schegg and Michael Ignaz
Schumacher. Published in
Journal of Tourism Futures.
Published by Emerald Publishing
Limited. This article is published
under the Creative Commons
Attribution (CC BY 4.0 ) licence.
Anyone may reproduce,
distribute, translate and create
derivative works of this article (for
both commercial and non-
commercial purposes), subject
to full attribution to the original
publication and authors. The full
terms of this licence may be seen
at http://creativecommons.org/
licences/by/4.0/legalcode 
of the tourism sector, as described in Hashim and Isse (2019). Services provided by these applications include: hotel reservations, airline ticketing, recommendations for local attractions or suggestions for disabled customers. The benefits of the deployment of such services are manifold, as it improves not only the user engagement and experience but also reduces access-to-service time and optimizes the use of resources (Ceccarini and Prandi, 2019; Ukpabi et al., 2019). Although many of these mobile/online applications are already successfully integrated into the workflow of mainstream tourism service providers, there is still a strong need for personalized and contextualized customer interactions.

Conversational agents (i.e. chatbots) are computer programs able to entertain a natural languagebased conversation with a human, and typically rely on Artificial Intelligence (Al)-based techniques (Arteaga et al., 2019). We can trace the origin of these technologies back to the 60 s when Joseph Weizenbaum developed ELIZA, the ancestor of modern chatbots (Weizenbaum, 1966). The goal of ELIZA was to simulate a psychotherapist engaging in fluent conversations. Thanks to different heuristics and workarounds, this early chatbot was able to obtain surprising results and avoid getting stuck, despite its limitations in knowledge and computational power. Although half-century elapsed from that revolutionary idea, chatbot technologies (CBTs) have only recently reached a satisfactory level to be widely adopted in real-life scenarios. Nowadays, we consider as chatbots (CBs) those computer programs able to interact with humans by using Natural Language Processing (NLP) or Al Markup Languages (leveraging on knowledge-bases composed of dialogue management rules and techniques for processing the user's input) (Ukpabi et al., 2019). These chatbots are mainly based on state machines, providing straightforward and easy-todesign interactions, although they are limited to rather simple conversations with human users (Calvaresi et al., 2019; Arteaga et al., 2019).

Specifically, in the tourism sector, CBs are commonly used to facilitate information and recommendation retrieval (e.g. opening hours of ski-lifts and restaurants (Hosseini, 2020)) and customer-care basic support (i.e. $85 \%$ of customer care in tourism is handled by chatbots/Albased systems (Ukpabi et al., 2019)). As an example, we can cite Mercure (AccorHotels) and its Facebook Messenger-based platform, which provides geo-referenced information to guests according to their position. Also, in the hospitality sector, Booking.com's service and support chatbot are widely available to English-language users, handling $30 \%$ of those customer questions automatically in less than five minutes. While these previous experiences provide evidence of the usefulness of chatbots in tourism services, it has not yet been assessed what is the impact of these technologies in a number of different aspects. Moreover, it is crucial to have a holistic understanding of the current state of the art, the challenges, opportunities and future perspectives in this area.

To address these issues, this work thoroughly analyzes the scientific relevance, industrial impact and concrete opportunities for CBs in the tourism, including its effect on future societies. To do so, we have conducted a semi-automated Systematic Literature Review (SLR), following a fully structured methodology. Following this methodology, we defined 12 research questions regarding different aspects upon which we analyzed existing works. This includes identifying what application scenarios have been addressed, who are the users of these chatbots, what type of services have been implemented, which technologies have been used, what are the benefits and drawbacks, etc. This extended revision of past works according to well-established criteria allows us to present an accurate picture of current research on this topic while pointing to the current needs and future directions. Furthermore, and complementary to the SLR, we have conducted a Focus Group (FG) study with industrial experts in the Swiss tourism sector regarding the potential of chatbots. This study allows us to understand with more detail the perception of chatbot technology for different stakeholders and how their expectations could be translated into innovation initiatives. Elaborating on the SLR and FG outcomes, we have identified use-cases based on the seamless thematic integration of tourists following social patterns, whose implementation can create remarkable "user experiences" and unveil concrete business opportunities. A concrete example based on social interactions through chatbots and dynamic 
provision of services by multiple tourism providers has been developed as a proof-of-concept $(\mathrm{POC})$, for which a survey has been elaborated in order to explore its potential and socio-technical possibilities.

The rest of the paper is organized as follows. Section 2 describes the systematic literature review, including a description of the methodology and the results. Section 3 elaborates on the study performed through a FG involving relevant stakeholders in the tourism domain in the region of Valais. Section 4 introduces the proof-of-concept implementation, along with survey results performed with the FG participants. Section 5 concludes the paper with a final discussion summarizing the results of the SLR, the FG and the POC.

\section{Systematic literature review}

The analysis of the literature performed in this study is rigorously structured and semi-automated to foster reproducibility (e.g. replicate the retrieval, selection and analysis processes). We present first the methodology (Section 2.1), including the definition of the research questions, and then we provide a complete description of the results (Section 2.2), in which we explain how each question is addressed in the studied papers.

\subsection{Methodology}

The SLR developed in this work extends our preliminary results (see Calvaresi et al., 2021) and relies on the original procedure for literature review presented in Kitchenham et al. (2009) and further adopted and adapted by Calvaresi et al. $(2016,2018)$ (see Figure 1).

Following this methodology, we started by formalizing the following generic free-form question: "How is the evolution of chatbots in tourism characterized?" In turn, according to the GoalQuestion-Metric (GQM) (Kitchenham et al., 2010), we have broken it down into the following structured research questions (SRQs):

SRQ1: Demographics. How time- and geographic-wise are the research efforts distributed? i.e. when (year) and where (the geographical indication of the scientific institute).

SRQ2: Abstraction. What is the abstraction level of the elaborated scientific contributions? e.g. what is the level at which the contribution is realized: conceptual $(C)$, prototype $(P)$ or tested $(T)$.

SRQ3: Application scenarios. Which applications/areas of the tourism domain have employed CBT-solutions? (e.g. hospitality, travel agency and transportation).

SRQ4: Recipients. Who are the users of CBT-solutions?

SRQ5: Desiderata. Which are the requirements standing behind the employment of CBT?

SRQ6: Goals. Which are the objectives set for CBT-solutions?

SRQ7: Services realized. Which CBT functionalities have been realized?

SRQ8: Services envisioned. Which CBT functionalities are desired and envisioned?

SRQ9: Technology. Which underlying technologies have been employed to realize the CBTs?

SRQ10: Benefits. Which advantages do CBTs provide? (from both user and provider standing points).

SRQ11: Drawbacks. Which limitations have CBTs shown?

SRQ12: Open challenges. Which open challenges concern the next generation of CBTs?

The semi-automatic research is characterized by contextual keywords and targeted keywords. In particular, the queries have been realized by combining the two sets listed below:

1. Contextual keywords: tourism + hospitality + traveling;

VOL. $\mathbf{m}$ NO. $\mathbf{m} 2021 \mid$ JOURNAL OF TOURISM FUTURES $\mid$ PAGE 3 


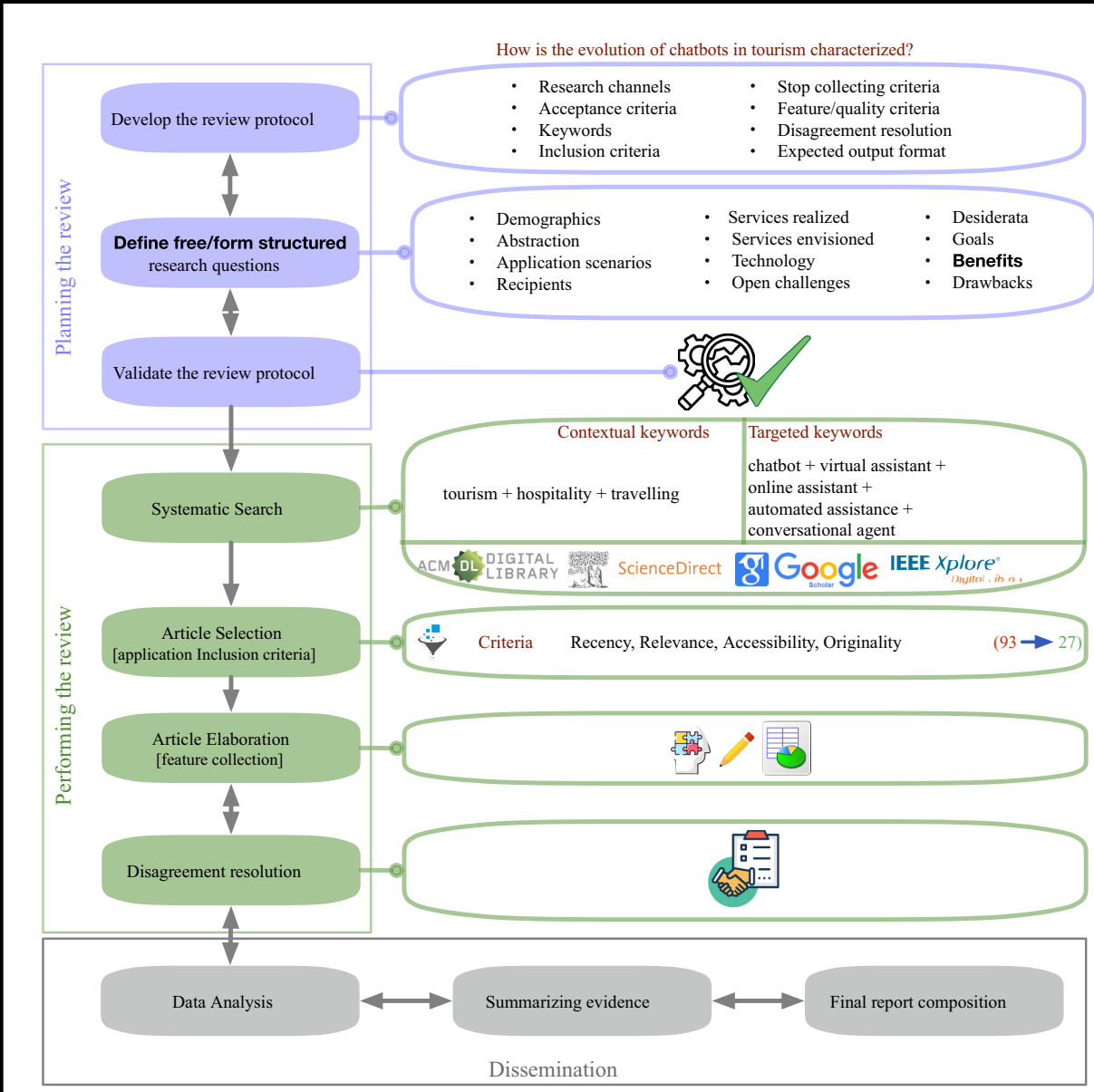

Source(s): Adapted from Calvaresi et al., 2016

2. Targeted keywords: chatbot + virtual assistant + online assistant + automated assistance + conversational agent.

The research of the articles has been conducted using the following sources: IEEExplore, Science Direct, ACM Library and Google Scholar. Ninety-three relevant papers have been initially collected. Performing a coarse-grained (parsing titles and abstracts), and successively fine-grained (briefly parsing the paper's content) examination, the primary studies to be elaborated have been reduced to 27 . In particular, the primary studies had to comply with the following criteria:

1. Recency (post-2016): We set 2016 as the starting year of the collection to identify the current trends and understand recent works addressing CBT in tourism. This is due to the scarcity and lower degree of maturity of previous efforts.

2. Relevance: The paper must confer relevant information and contribution to the tourism sector. Therefore, scholarly papers without a clear and explicit link to the tourism domain have been excluded.

3. Accessibility: To be included, the content of the article should be accessible via one of the portals mentioned above.

4. Singularity/Originality: Duplicate papers or papers having an extended follow-up version are not included. Only the complete version is included. 


\subsection{SLR results}

This section summarizes the results of the systematic literature review, grouping them according to research questions formalized above. We analyze the reviewed papers according to each of those dimensions and provide details about specific works when necessary.

2.2.1 SRQ1: Demographics. The paper selection and elaboration have been conducted in late July 2020, which justifies that only 11 papers were collected in that year. Nevertheless, the trend projection suggests a growing interest from the scientific community. This trend is expected to grow also in other communication channels (e.g. tourism industry news and press releases), although these fall out of the scope of this review. The paper distribution per country is shown in Figure 2(b), reflecting the worldwide interest for chatbot technologies in the tourism domain.

2.2.2 SRQ2: Abstraction. Most of the papers propose practical and tested solutions (16 studies), five studies present prototype-level systems and six focused solely on conceptual contributions. This distribution reflects the predominantly practice nature of CBT initiatives in the tourism domain. Nevertheless, on many occasions, the deployed prototypes or tested systems sacrifice technical novelty in favor of functional but simplistic implementations. Even in the case of tested solutions, the methodology is heterogeneous, with important variations in terms of the number of participants, a degree of involvement, time of use, etc.

2.2.3 SRQ3: Application scenarios. Most of the studied scientific contributions are linked to applications for hostelry, airlines, travel agencies and related sub-domains (20 studies). Four promote specific areas or cultural heritage sites (e.g. CBs for promoting the city of Manta in Ecuador (Arteaga et al., 2019) and Pompeii's archaeological park in Italy (Lombardi et al., 2019)). Moreover, one study focuses on medical tourism, developing a CB to identify medical drugs available in the visited locations which are equivalent to the ones sold in the tourist's origin location (possibly unavailable).

The primary concern of all the elaborated studies is to smoothly handle high volumes of customers 24/7 (Ukpabi et al., 2019). Moreover, to simplify the CB-based dynamics for the end-users, the studies investigated and identified new ways to drive the user through a booking (Popesku and Zlatanov, 2019), determined the most opportune answers (Quicktext, 2019a) and the most opportune tone to approach the user in given conditions (Hu et al., 2018). In turn, the focus goes to satisfying the functional requirements indicated by the service providers (i.e. enhance the system performance (Bozic et al., 2019), automating and testing new functionalities (Bozic et al., 2019))

Figure 2 (a) Number of papers per year (b) Number of papers per country

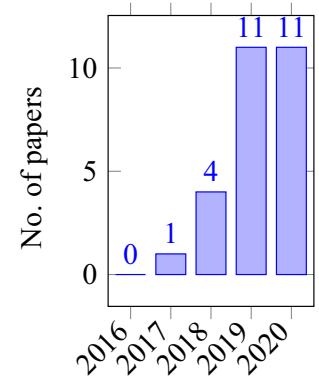

Number of papers per year

(a)

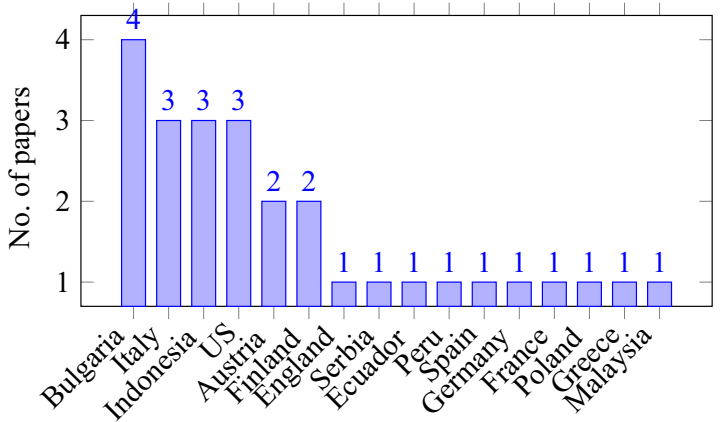

Number of papers per country

(b) 
and improve the data collection (i.e. preferences and feedback (Hu et al., 2018; Ukpabi et al., 2019)). As we can see, some of these aspects are orthogonal to the domain of application and show the potential of generalization. However, most of the developed prototypes are designed according to application-specific criteria, even relying on ad hoc mechanisms if necessary.

2.2.4 SRQ4: Recipients. Although CBs are intended to interact with any typology of user, Millennials and Generation $Z$ have been determined as primary recipients of the chatbot technologies. This is mainly due to the IT literacy level of this segment of the population, who are often already users of major messaging platforms such as Facebook or Telegram. Moreover, these users already have the motivation to use automated booking and searching tools while being targeted by CB campaigns over social media (Amalia and Suprayogi, 2019). Nevertheless, it cannot be overlooked that, for older users, the interaction with chatbots can also help reducing time spent on booking and reservation purposes.

2.2.5 SRQ5: Desiderata. The requirements elicited from the primary studies can be classified into four main categories: financial, technological, socio-technical and socio-management.

Implementing effective and competitive CB-based solutions requires considerable financial investments (Buhalis and Yen, 2020), possibly spanning from $\$ 30.000$ to $\$ 150.000$ (Lukanova and llieva, 2019). Moreover, to satisfy complex user requests, some CBs need to gather data from services such as Avis, Uber, IBM Watson, Google Dialogflow and Google Maps, resulting in an increase of the costs (Arteaga et al., 2019). Therefore, implemented chatbots should justify the investment through significant and measurable economic benefits.

The technical knowledge required to realize CBs is remarkable, and it requires to model user and system dynamics (Bozic et al., 2019), identify and design the proper architectures (Arteaga et al., 2019), model and automate processes and tests (Bozic et al., 2019; Melián-González et al. , 2019), and model and implement data collection, compliance, storage and protection (Arteaga et al., 2019; Hosseini, 2020; Ruf et al., 2020). At this point, it is important to consider to what extent the reuse of existing CBTs is enough and if a dedicated development is necessary (given the required technical skills to realize it).

Enabling semantic interactions is a socio-technical task demanding a remarkable effort. For example, enhancing FAQ-related interactions from a basic structured and bare-metal protocol to a more dynamic, explicative and user-friendly process is not negligible (Buhalis and Yen, 2020; Amalia and Suprayogi, 2019). These developments usually require the usage of co-creation methodologies that facilitate the design of effective communication and interaction strategies. Customer care vastly benefits from features such as tone identification and definition, classification and representation of the context (i.e. via context dimension tree) (Lombardi et al., 2019; Clarizia et al. , 2019). For example Hu et al. (2018), highlighted the significance and impact of using different tones in the context of social media customer care. In turn, solving ambiguities, data and error handling (Hosseini, 2020), monitoring and evaluating the chatbot effectiveness/efficiency, and seamlessly hand the conversation from the chatbot to a human operator if stalling (Hosseini, 2020) are gathering the researchers' attention (Bozic et al., 2019).

Procedural and management qualifications, as well as user-based analysis to be conducted for a more personalized experience and marketing, compose the socio-management requirements (Lombardi et al. , 2019; Ivanov, 2019; Ivanov and Webster, 2017). For example, relevant changes to the system can be notified to the concerned personnel and via CBs, which at the same time can trigger maintenance and updates of the service documents (Ivanov and Webster, 2017). Furthermore, by revising some paradigms, the workforce can be trained or tested on the latest updates of their systems. By doing so, their concerns and resistance to the changes might be softened (Ivanov and Webster, 2017). From the marketing perspective, developing a communication program to inform customers, suppliers and other stakeholders via $\mathrm{CB}$ is becoming a priority requirement (Ivanov and Webster, 2017). Finally, data analysis (post-marketing

PAGE 6 | JOURNAL OF TOURISM FUTURES $\mid$ VOL. $\mathbf{m} \mathbf{m}$ NO. $\mathbf{m} 2021$ 
or about the user behavior) is crucial to better define, understand and possibly predict their behavior (Melián-González et al., 2019).

2.2.6 SRQ6: Goals. For each of the papers analyzed during the review process, the domain of application has a notable influence on the goals of the CBs. For example, 9 industry-related papers mainly focus on the current state of CBTs in the tourism industry, and 11 purely Academic-related papers focus on technical aspects and development of the chatbots (including front-end and back-end functionalities).

Nevertheless, a common objective in almost all these works is to provide continuous and automated support, thus guaranteeing extended access to information at all times and a lower cost. This translates, for example, to promoting 24/7 availability to tourists vising cultural and heritage sites for the city of Manta in Ecuador (Arteaga et al., 2019) and for Pompeii's archaeological park in Italy (Lombardi et al., 2019).

Chatbots can also convey local information through social media, as in Amalia and Suprayogi (2019), where the Messenger platform is used to provide continuous interactive tourism information about Yogyakarta. The provision of incremental or new functionalities tries to keep the pace of the quick-evolving technologies to enhance the user experience. For example Hu et al. (2018) created an original tone-aware CB that generates toned responses. Bozic et al. (2019) automated the testing of CB's functionalities. Clarizia et al. (2019) introduced a CB relying on a context-aware system able to recommend contents and services to increase the promotion of cultural heritage. Finally Ruf et al. (2020) realized a companion CB to help travelers decoding medical drugs sold in the host country, linking them with the corresponding trade name sold in the traveler's home country.

2.2.7 SRQ7: Services realized. The elaborated studies present services that can be classified as technological and socio-technical.

From the technological perspective, back-end functionalities captured most of the scientific effort. For example Sano et al. (2018) focused on mining and manipulating the acquired data; Bozic et al. (2019) focused or automating the CB testing via Java-based implementations that automatically parse plans and generate concrete test cases at run-time; Arteaga et al. (2019) realized an architecture to extract users' intents and expectations searching for text patterns in the users' messages. Finally, other services to mention are hotel-related forecasting (i.e. tourists arrivals, demand and hotel occupancy) and analyzing the impact of online reviews on hotel performance to offer the provider a better and clearer vision in the long run (Ivanov, 2019). From the user perspective, CBs interfaces are mainly composed of the chat showing the messages exchanged and the keyboard (or an interaction menu prizing simplicity and efficiency). Nevertheless, a few applications such as (Kasinathan et al., 2020) opted for reserving an important portion of the screen/window for a cartoonized CB, in the case of Figure 3 a flight assistant.

From the socio-technical perspective, the analyzed functionalities can be related to service management (e.g. booking a room or answering FAQs (Buhalis and Yen, 2020; Quicktext, 2019a; cvent, 2019)), ordering meals or drinks (phocuswire, 2018), controlling the room temperature, lighting, taxi booking and itinerary planning (phocuswire, 2018; Chrysovelidis, 2020), and identifying a corresponding medical product from the user's home market (Ruf et al., 2020).

In Buhalis and Yen (2020) the CB can solely communicate to the client pre-arrival, throughout their stay and post-checkout. In Hu et al. (2018) the CB can generate toned responses to user requests based on their humor using the seq2seq model implemented with recurrent neural networks (RNN), such as the Long Short-Term Memory (LSTM) or the Gated Recurrent Units (GRU) model. In Lombardi et al. (2019) the CB can provide necessary information to offer a better touristic experience. Finally, in Clarizia et al. (2019) the CB can even adapt the user interface according to the visitor's backgrounds to enhance the personalization and quality of experience.

VOL. m. NO. ma $2021 \mid$ JOURNAL OF TOURISM FUTURES $\mid$ PAGE 7 


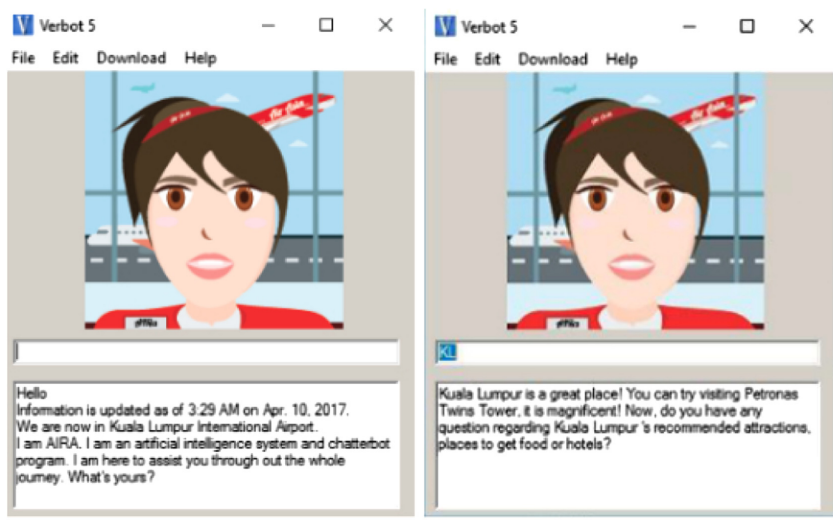

(a)

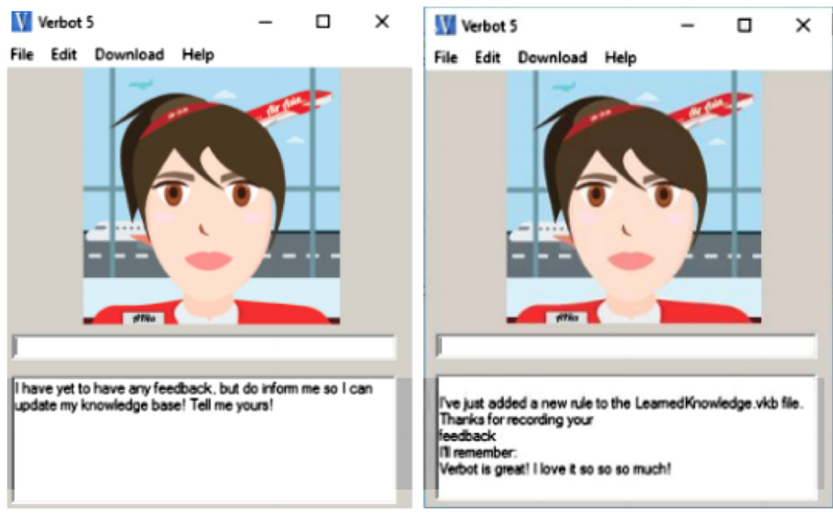

(b)

CBs' marketing and sales-related functionalities are crucial. To this end (Popesku and Zlatanov, 2019) and (Arteaga et al. , 2019) created CBs able to provide personalized travel recommendations of touristic sites and attractions; Amalia and Suprayogi (2019) promoted marketing campaign based on consumer involvement; phocuswire (2018); conceived promotion/suggestion of special dishes; Ivanov and Webster (2017) focused on promotion and reminding the services available in the hotel, going beyond the classic guests-greetings at check-in/out from the hotel.

Finally, some CBs can leverage on analysis tools, allowing the service provider to extract aggregated and derived users' information (e.g. intents and expectations) to elicit the users' preferences (Arteaga et al., 2019), to learn from previous users' choices to predict further interests (Clarizia et al. , 2019), to forecast tourism arrivals, demand and hotel occupancy and to analyze the impact of online reviews on hotel performance (Ivanov, 2019).

2.2.8 SRQ8: Services envisioned. The functionalities that have been conceived, but not designed/ implemented yet, are socio-technical and technological.

The envisioned socio-technical functionalities primarily concern the personalization. For example, tailoring guests' stay and experience via voice commands integrated in the CB (Buhalis and Yen, 2020), enhancing and extending the level of interaction of the CB's personal guide-related tasks managing the overall stay of the tourist (Arteaga et al., 2019). Other examples are direct 
connections between the smart hotel rooms' amenities/services and the CB (exposing the same level of configuration); Buhalis and Yen (2020) implementation of emotions-based mechanisms to develop proactive CB; Buhalis and Yen (2020) (increasingly leveraging on Al and ML) realized trainable/trained CB to learn users' behavioral styles to be mirrored. Moreover, CB might behave according to specific styles, possibly identifying given brands (Hu et al., 2018), increase the possibly heterogeneous data-sources (e.g. feedback and emotional/psychological) and formats to enrich the CB knowledge and services to face more complex environments (Clarizia et al., 2019).

The envisioned technological functionalities revolve around automating mechanisms and behaviors, improving testing coverage and transparency targeting more generalizable approaches (Bozic et al., 2019) and studying CBs' principles and pillars to enable a deeper understanding of the technologies and their potential evolution to meet modern and future needs (Hosseini, 2020).

2.2.9 SRQ9: technology. A plethora of technologies has been employed in the chatbots released so far. Many systems adopt stand-alone back-ends (mostly relying on Python libraries) entirely developed by the commissioned service provider. However, in more complex cases, CBs are integrated with existing third-party solutions, especially back-end platforms/services such as IBM Watson (phocuswire, 2018). Concerning the front-end, they use either customized implementations or rely on existing platforms such as Telegram (Dyrkolbotn et al., 2018) and Facebook messenger (Popesku and Zlatanov, 2019; Arteaga et al., 2019; Amalia and Suprayogi, 2019; Hosseini, 2020).

2.2.10 SRQ10: Benefits. Multiple advantages can be acknowledged to the analyzed CBs (both time- and quality-wise). Usually, customer satisfaction revolves around the perception of time, which is even more emphasized in the tourism sector. CBs are perceived as a 24/7 working concierge always available and providing instant support/solutions (Buhalis and Yen, 2020; cvent, 2019). CBs reduce and simplify the human-machine interaction process (i.e. $\sim 80 \%$ of all customer requests are automatically processed, delegating to the human personnel only the remaining 20\% (Quicktext, 2019a)). CBs can usually cope with numerous, simultaneous and personalized conversations - only limited by the hosting machine (Buhalis and Yen, 2020). The services provided have constant quality levels, which are not affected by common employeerelated risks (i.e. strikes, discrimination, quitting the job with no notice, showing negative emotions, shirk from work, and getting ill (Buhalis and Yen, 2020; Ivanov, 2019)). To date, despite explicit or implicit ethics implementations (Bozic et al., 2019), no CB on the market has raised complaints about its fairness or misconduct. Surprisingly, some tests have indicated that the responses generated by the CBs have been perceived as more empathetic than those provided by human agents, thus raising customer appreciation (Hu et al., 2018).

Indeed, CBs have received positive feedback for the dynamic dissemination of heterogeneous information, services or narrative content (textual and multimedia), which enabled their integration and adaption with respect to the users' needs and dynamic behavior-rarely raising complaints about the respect of the users' privacy (Clarizia et al., 2019; Amalia and Suprayogi, 2019; phocuswire, 2018). Although quick, some interactions may require complex computations. Nevertheless, such sophisticated analysis converge in a human-reasonable amount of time, enabling a prompt understanding of the customer requirements and prompt predictions for more accurate replies and overall interactions (Melián-González et al., 2019). Finally, the financial benefits provided by the CBs are tangible. For example, savings employees' time from tedious and repetitive tasks (Ivanov and Webster, 2017) -therefore contributing to reduce personnel demand and staff workload (cvent, 2019; Lukanova and llieva, 2019), automatizing the advertising activities, recording growth in sales and, overall, increasing the brand's value (Lukanova and llieva, 2019).

2.2.11 SRQ11: Drawbacks. Elaborating the primary studies it is possible to group the limitations into three categories, i.e. user-, provider- and system-related.

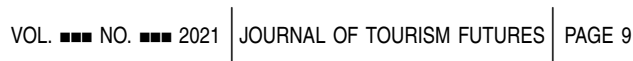


Concerning the providers, small businesses can rarely afford the design, development and maintenance of CBs. Indeed, as mentioned above, costs can range from $\$ 30,000$ and up to $\$ 150,000$ or even more if more complex analysis and integration with third-party services are required (Popesku and Zlatanov, 2019; Lukanova and llieva, 2019; Ivanov, 2019). Using a CB as the only way to contact the service provider can represent a single point of failure of the communication, possibly raising frustration and delusion in the user if misunderstanding and unfruitful loops occur.

The limitations extracted from the primary studies concerning the chatbot itself are the incapability of processing complex information, thus resulting in scattered and artificial/unnatural interactions, looping on inappropriate suggestions and the difficulty to interpret (in)satisfaction (e.g. sarcasm) still foster reluctance on the employment of these technologies (Buhalis and Yen, 2020). The users might also be required to share private information about their complaints or financial situation (considered sensitive), which raises the fear of having them compromised or, if misinterpreted, misused (Buhalis and Yen, 2020). Moreover, the lack of creativity, involvement and personal touch are recurring critics affecting the elaborated solutions, especially in case of request misinterpretations (Buhalis and Yen, 2020; Bozic et al., 2019). Therefore, the scientific community still considers the human factor essential and unavoidable in the near future to support/supervise the CB (Ivanov, 2019).

Finally, CBs can be perceived as threats. For example, from the "powerless" misunderstood user or the service provider employees (i.e. help-desk) who see their work positions endangered (Buhalis and Yen, 2020; Melián-González et al., 2019).

2.2.12 SRQ12: Open challenges. As with the advantages, the open challenges are mainly provider-, user- and system-related. Within the chatbot dynamics, the human user is central. Nevertheless, CBs still struggle with lexical and semantic ambiguities (Ukpabi et al., 2019). Therefore, the most impelling challenges are user-related. For example, to align the CB with the users' perspectives, to prevent user's uncertainty and resistance (Buhalis and Yen, 2020), to determine users' perceptions via NLP (Chaves, 2020), to promote clarity and wording to match or compensate the users' feelings (Melián-González et al., 2019), to pace the conversationchoosing number and length of the words - (e.g. longer words are more calming and associated with positive emotions) (Melián-González et al., 2019), and avoiding bothersome repetitions (Ukpabi et al., 2019). However, the communication style of the human users changes if they know the recipient of their messages (the interlocutor) is a CB (Melián-González et al., 2019). Thus, to understand to which extent a designer should chase the human-like feeling rather than a more clear/structured interaction is still an open question (Lombardi et al., 2019).

From the provider perspective, the main open challenges are to find the right trade-off between chatbot- and human-delegated tasks (i.e. managing the loss of jobs (Buhalis and Yen, 2020)) and enabling knowledge sharing (Buhalis and Yen, 2020). In turn, can the realization of an effective business plan, which must generate a considerable return of interest (ROI), be considered a CBdelegable task (Buhalis and Yen, 2020). Finally, considering the nature of the primary studies (more tourism-centered than technology-oriented), the system-wise open challenges have not been fully explored. Indeed, the challenges identified by the elaborated papers are quite broad and focus on data extraction and data representation (Hu et al., 2018), ensure data correctness and bias-free (Amalia and Suprayogi, 2019), and Al-related functionalities and features (Quicktext, 2019a).

\subsection{SLR analysis and discussion}

Modern chatbots have made substantial progress beyond the seminal vision proposed back in the 60s (Popesku and Zlatanov, 2019). Although the original challenge was to deceive the human user on the nature of the interlocutor behind the chat, nowadays, the focus shifted more toward the interaction and the more exchange of information. Over the years, CBs achieved a considerable degree of automation and efficient execution of well-structured tasks/interactions (e.g. ordering

PAGE $10 \mid$ JOURNAL OF TOURISM FUTURES $\mid$ VOL. $\mathbf{m}$ NO. $\mathbf{m} 2021$ 
meals and booking flights). However, CBs do not master sophisticated conversations yet. Indeed, misunderstandings and repetitive loops may cause distress and frustration, generating skepticism about a given CB or the technology itself. For example, the Japanese hotel Henn-na has heavily invested to offer its guests a futuristic staff mainly composed of robots. Nevertheless, in 2015 they had to "fire" $50 \%$ of their robotic workforce. The failure of the project can be adduced to the broken promise of costs and employees' workload reduction. Additionally, several tourists reputed those (ro)bots irritating which occasionally even failed to satisfy simple requests (Quicktext, 2019b).

Overall, the most common CBTs rely on state machines (rule-based interactions) and on standardized menus (thus removing the need to produce/parse custom verbal text -i.e. via NLP) (Ivanov, 2020). Although it limits the expressiveness of the conversations remarkably, this strategy reduces the system complexity and the likelihood of errors and misunderstandings.

To perform an in-depth analysis of both provided data and the human interlocutor's profile, the CBs' engine needs to be more sophisticated. Advanced Al-based NLPs are not limited to understanding what the user is saying, but also strive to understand tone, mood, etc., enabling ML-based predictions. ML approaches require a large amount of data to provide satisfactory results. To date, such processes are still laborious and human intense. Hence, reducing human involvement in data extraction and pre-processing is a common priority. Notwithstanding, having a deeper understanding of a tourists' interests, preferences, financial capabilities and personality can exploit ML predictions for more tailored assistance/information and, more importantly, shaping future interactions. Indeed, e-communications outperformed conventional methods, forcing hotels and the tourism industry in general to evolve their systems embracing new messaging platforms and social media. Platforms such as Facebook and Telegram have remarkably invested in developing APIs for the deployment of CBs. For example, Facebook Messenger counted 66.000, 100.000 and 300.000 active chatbots in 2016, 2017 and 2018 respectively (Ukpabi et al., 2019; Machine, 2018). Recently, the users of the Telegram platform skyrocketed (300 million of CBs in 2018). The high-quality APIs and services of these platforms are attracting an increasing number of businesses (Morze et al., 2017). The Slack platform provides an early-version of CBTs, allowing the configuration of autoreplies and personal-tasks automation (i.e. reminders). However, the bot does not support conversations (Haque, 2019). Finally, to date, Whatsapp is still relatively behind (APIs development phase) w.r.t. the other big competitors (Jindal et al., 2020). The investments of these ICT colossi reflect the hype for $\mathrm{CBs}$ and the possible market opportunities. Indeed, several brands are deploying their own CB into these platforms (i.e. Lysa, CNN and Pizza Hut (Popesku and Zlatanov, 2019)).

Many industries operating in the tourism sector see chatbot technologies as a challenge to undertake mandatorily (a new dimension for an already harsh competition). Thus, trying to impose the brand-characteristic traits in this new technological competition. For example, hotels are investing in providing virtual concierges with the most innovative functionalities off the shelf. CBs can both strengthen or crush customers' satisfaction, hindering their loyalty. Creativity, originality and efficiency are crucial in this new quest.

Given the competition in the market and the technological fast pace, rule-based chatbots risk becoming outdated quickly. Thus, CBs initially representing an advantage might backfire if the brand/service provider does not invest in its evolution to match the users' expectations. Indeed Hu et al. (2018), confirms such a transitional trend, highlighting the strategical evolution from rulebased systems to fully NLP-based CBs, setting as an upcoming challenge the inclusion of empathy and social engineering (Hadnagy, 2010). Such a study anticipates the benefits of this direction in terms of user satisfaction, which is also proven by the KLM Royal Dutch Airlines' novel CB that supports the tourists in packing for their trip, just knowing destination, date and trip length (Popesku and Zlatanov, 2019).

Summarizing, CBs' 24/7 data availability and menu-based interactions are a must and extremely appreciated. However, to date, those represent only the entry-level features that modern CBs must provide. Data integration, storage and manipulation are challenges that will continuously evolve, driven by the goals such as (1) anticipating/predicting the user, (2) content- and tone-aware 
debating capabilities and (3) personality traits codification (possibly embracing the brand etiquette and overall style). In tourism and hospitality, the development of CBs' front-end and back-end functionalities represents a remarkable investment and strategy shift. Finally, the lack of ethical considerations raises a few concerns. None of the elaborated studies has addressed the CBs' behaviors and/or data-management plans (DMPs) from an ethical perspective. Given the sensitive nature of the data handled, this is a great limitation of the current studies, which require an impelling intervention.

\section{Focus group study}

Although the information and insights extracted from the literature review provide valuable knowledge about the use of chatbots in the tourism sector, a more detailed connection with realworld stakeholders and their perceptions/inputs is missing. Therefore, in this section, we describe a FG study conducted with tourism experts to complete the picture. FGs have been used to support qualitative research analysis in the past decades by enabling interactive interactions among individuals with similar, yet possibly heterogeneous, backgrounds. The participants of the FG mainly belong to the Canton of Valais area (one of the most Touristic regions in Switzerland). Among the participants, we included CEOs, directors and marketing officers of regional tourism organizations and hostelry associations, as well as tourism university professors and researchers.

The applied methodology is described in Section 3.1.

\subsection{Focus group methodology}

Due to the pandemic situation at the time of writing, the FG has been run virtually and recorded for further analysis (via an agreement with the participants). The FG is composed of five main activities:

1. Kick-off

Introduction of the subject, setting the common ground and self-presentation of the participants.

\section{Question-driven discussion}

The questions listed in Table 1 have been used to drive the discussion and stimulate the interactions, which, in turn, have been left free to evolve.

\section{Run-time data collection}

Penciling in main points and unveiled connections during the meeting.

\section{Final wrap-up}

\section{Table 1 Questions leading the focus group discussion}

ld Questions

FGQ1 Do we all know what a chatbot is?

FGQ2 Which tourism services can be delegated to a chatbot?

FGQ3 What can push tourism workers to desire a chatbot?

FGQ4 What do you think Al can do for the tourism industry (especially if coupled with chatbots)?

FGQ5 Which are the strings attached to deploying a bot? (advantages and disadvantages)

FGQ6 What would you be able to ask a chatbot? (as a customer/service recipient)

FGQ7 How do you envision the data collected by a chatbot? What would you be able to do with them?

FGQ8 What is your opinion on the legal and ethical aspects regarding the data collected?

FGQ9 How can we humanize the conversations to make them pleasant and guide the dialogue appropriately?

FGQ10 What can a chatbot perform better than a human agent?

FGQ11 What must a chatbot NOT do? 
A quick run through the meeting notes to summarize certainties, doubts and envisioned challenges outcomes of the FG.

\section{Off-line analysis}

Manual transcript and elaboration of the discussion to improve the formulation, mapping the topics and formalize the overall understanding.

\subsection{Focus group results}

Figure 4 graphically summarizes the topics and related connections that emerged from the focus group.

Either as a user or service provider, every participant has dealt with a chatbot at least once. The heterogeneity of the experiences leads to a diverse understanding and perception of what a chatbot is. Nevertheless, only some of them really understood the potential of chatbot technologies. In line with the SLR findings, the magnitude of the economic investment has been pointed out during the FG. Besides top-destinations such as Zermatt and Leukerbad, where about $40 \%$ of the hotels are $4 / 5$-star, the low-price and dimension hotels/facilities are predominant in most tourist sites in Valais. Moreover, the experts have highlighted that the management of many touristic infrastructures is often family-owned, which justifies the limited investments in CBT.

Nevertheless, all the experts agreed on the idea that "a CB is a must-have". For example, they see a great need for semi-automated functionalities such as information pre-filtering to facilitate the handover to human operators via systematic/structured interactions. On the one hand, this view apparently downsizes the role of CBs, due to the limited knowledge shown by some experts about their potential. On the other hand, it raises awareness about the relevance of habits. For example, Millenials and Z-generation can fluently interact with a CB, while older generations might feel more confident having contestable communication proofs (e.g. emails) or being reassured by a human voice (e.g. via front-desk or phone call). Nevertheless, if well designed, tourists from almost every age range can use a CB and experience a positive involvement.

The typology of information provided is also extremely relevant to discriminate whether or not it is expected/wanted from a CB or not. In particular, some experts referred to the information provided by the $\mathrm{CB}$ as redundant, already possibly available on illustrative guides or websites. If the traits of

Figure 4 Graphical representation of the topics distribution and connection

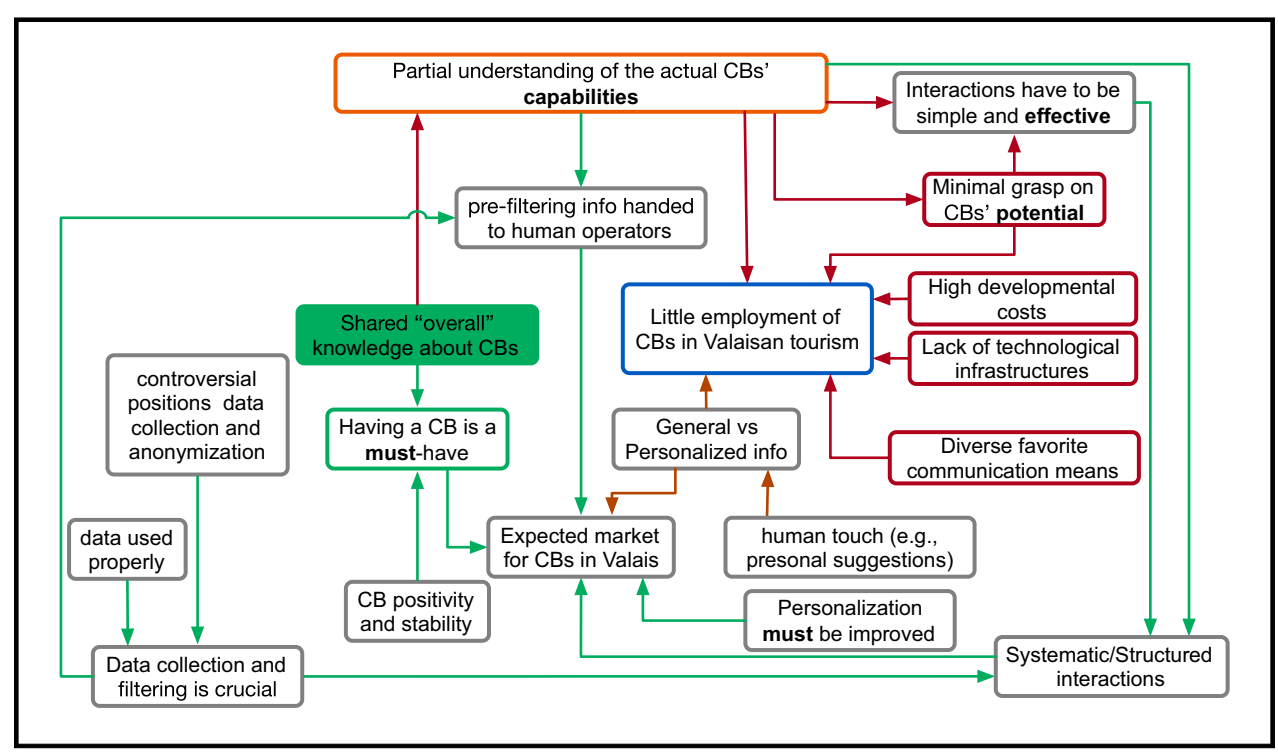

VOL. $\mathbf{m}$ NO. $\mathbf{m} 2021 \mid$ JOURNAL OF TOURISM FUTURES $\mid$ PAGE 13 
the information get more tailored, then most of the experts would agree on the need for CBs. Nevertheless, a minor group still raised some resistance, reinforcing the importance of the personal (human) experience enriching the suggestions and the overall interactions. Some experts indicated that although the human touch is always welcomed, CBs can sometimes be more empathetic (i.e. CBs have no bad days) with respect to human personnel. To date, the personal recommendations based on real personal experiences have no Al competitor. Nevertheless, tourism CBs can deeply benefit from profile-learning techniques powering $\mathrm{CBs}$ in eHealth and assisted living domains (Calvaresi et al., 2019).

Finding the trade-off between simplicity and personalization becomes paramount importance and raises the question: how can the interaction be simple and personalized at the same time? Having a simple interaction means limited messages (henceforth data) exchange. If not from the current chat, how can the CB get enough data to offer a satisfying personalized experience? The data collection and profiling go beyond what happens in the chat at run-time. For example, offline training is needed to support the CB in inferring parameters such as context, user behavioral models and profile to simplify the communication.

On the one hand, collecting data raised a significant discussion in the group, with a few experts concerned about consent management and user privacy. On the other hand, some experts reported the scarce care the users generally have about their data and privacy. In particular, they referred to internal studies about the user behaviors on the websites of their businesses, where besides the updated policies about websites cookies, roughly $85 \%$ of the users have given consent without even opening the descriptive pages. Although awareness about ethics and privacy in CBs scenarios has not peaked yet, all the experts agree that the quality of the services must not come at their expense. Overall, according to the experts' experience, tourists do not mind having their data collected to have better services, as long as their identities remain hidden (anonymous data), so that they cannot be traced back in any way. Nevertheless, there are more strings attached with respect to what regular tourists and tourism experts perceive nowadays.

The discussions raised during the FG have highlighted that the industrial experts' viewpoints are committed to conventional dynamics and narrowed by their technological knowledge. As summarized above, the findings obtained with the FG confirmed and extended the results obtained with the SLR. Surprisingly, CBs have not been related (not in the SLR nor in the FG) to the need for supporting the social needs of tourists and optimizing the limited facilities and guides/ trainers of the touristic destinations. In addition, the hidden economical benefits of having seamless and dynamic tourists integration via CBs have been overseen. As proven in previous works, the social dimension can play a pivotal role in unveiling new business opportunities. Therefore, we have decided to raise that possibility, analyzing the reaction of the experts. After a short moment of silence following our suggestion, the experts manifested their curiosity on exploring this idea further, which is reflected in the following section.

\subsection{The social dimension for tourist, service providers and chatbots}

Chatbots have a potential that goes beyond the imagination of many service providers in the tourism sector, who reductively see them as mere state machine-based information providers. To date, no existing system, prototype or theoretical design conveys multiple tourists, service providers and chatbots in dynamically generated chats (e.g. thematic and per time-window). The idea stems from the awareness that touristic destinations have several, yet limited, resources (e.g. sports facilities, guides or trainers) that often are unsystematically contended by the guests. Nevertheless, the offer and the tourist involvement in such activities can be boosted, if planned per time. Indeed, deploying "means" to seamlessly stimulate, aggregate and tunnel the tourists' activities can increase their satisfaction, unveiling unexpected possibilities and generating business opportunities. For example, chatbots could gather athletes willing to play the same sport in a given time-window (their stay) in a given location, but missing adversary(ies)/partner(s) both last minute or even before the vacation is actually started. Such a gathering is not limited to the 
guests of a given structure, and it could involve visitors/residents of the given area. Indeed, we envision that enabling the interaction among tourists (possibly including people living in the area), facilities and dedicated personnel (e.g. instructors) would reshape the decision-making dynamics, possibly even impacting fundamental choices (e.g. picking the facilities or even the locations).

Figure 5 schematizes the envisioned dynamics of CBs for future social tourists.

On the one hand, organizing the group activities ahead of time (e.g. during the planning of the vacation) may secure the availability of trainers, guides or other athletes per time and maybe with more advantageous costs. On the other hand, it is beneficial even for tourists with less planning attitudes, eliciting last-minute opportunities almost effortlessly. Many types of activities might benefit from such an organization. For example, concerning the Swiss offers, we could mention glacier group excursions, sports classes, team sports and even cultural site visits. Moreover, some activities might require gathering/renting the necessary materials (e.g. skis, sticks, ropes or rackets). To ensure a seamless organization, the specific bot in charge of a given activity in a given time window can be involved in the group chat of equipment providers to ensure a seamless organization. Eventually, if necessary, this may include even autonomously booking sports facilities (fields/courts) per time.

After having sketched this idea in the FG, we have decided to realize a PoC, which, a few days after, has been shown to the experts participating in the $F G$ and individuals unaware of the $F G$ discussion. The PoC showed some practical possibilities in real-world scenarios, which concluded with a structured survey.

\section{Chatbots for future social societies of tourists - proof-of-concept}

To realize the PoC, we relied on the Flow.ai framework (Flow.ai, 2020). Such a platform is ideal for the fast development of both simple and sophisticated solutions. In particular, the basic behaviors can be set up as separate modules (named activities) whose basic dynamics can be realized through "drag-and-drop" functionalities in the behavioral flowchart. The basic behavior of such components can be extended by calling external scripts and services.

\section{Figure 5 Stakeholders and dynamics of future CBs for social tourists}

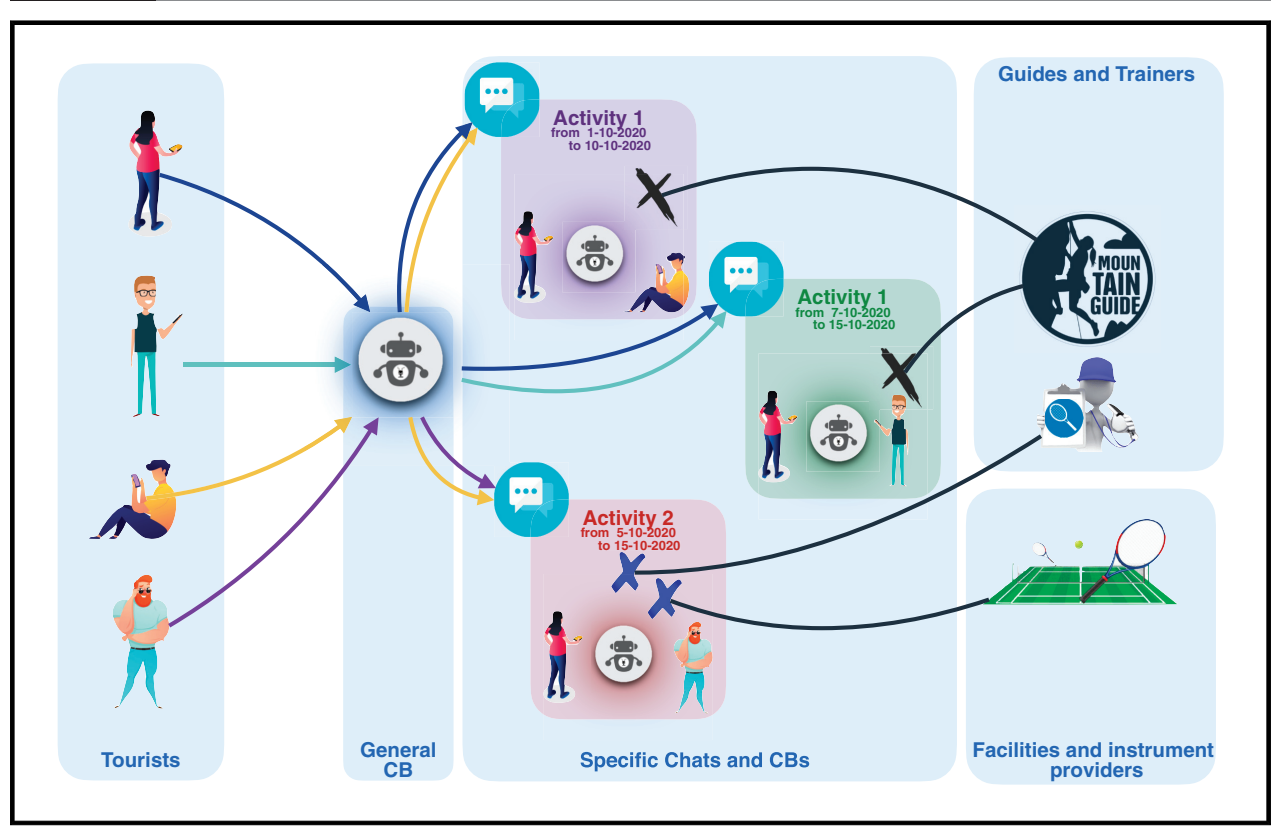

\begin{tabular}{l|l|l} 
VOL. $m$ NO. 2021 & JOURNAL OF TOURISM FUTURES & PAGE 15
\end{tabular} 
For this PoC, we set the following requirements:

\section{R1: Basic user profiling}

R2: Booking a stay in the desired destination

R3: Reservation of activity in the desired location (even without a hotel booking)

R4: Redirection of users to an activity-based group chat according to the activity's location, user's activity level and the period of time

R5: Overview of the bot functionalities (i.e. menu)

R6: Consent-based interaction

According to the requirements, we drafted the following basic functionalities.

1. Greetings: To initially "wake-up"/trigger the bot to begin the conversation, the user has to send a message. The bot has a basic mechanism to recognize, assume and learn new greeting terms such as Hi, hil, Hello!, hello, Yo, Coucou, Hey, etc. (see Figure 6). In turn, the bot asks for minimal personal information (i.e. name, surname and email) to briefly profile the user (see Figure 7a). Such information is only temporarily stored (cached) for the duration of the interaction. Nevertheless, if the user does not wish to disclose them, the interaction is interrupted (see Figure 7b).

2. Main menu: the completion of the profilation enables access to the "main menu" summarizing the functionalities of the bot in the presented PoC. Moreover, such a menu is reachable by asking (typing) help to the bot at any time (except if answering a specific question). The details of the functionalities provided by the menu follow.

3. Book a destination: Three simple steps compose this functionality such as (1) picking among a selection of destinations proposed via a slider within the chat (Figure 8a), (2) indicating number of guests, period and budget night (Figure $8 b$ ) and (3) selecting the hotel from a selection matching the user's budget (Figure 8c).

\section{Figure 6 Unclassified greetings triggering the bot to begin the conversation}

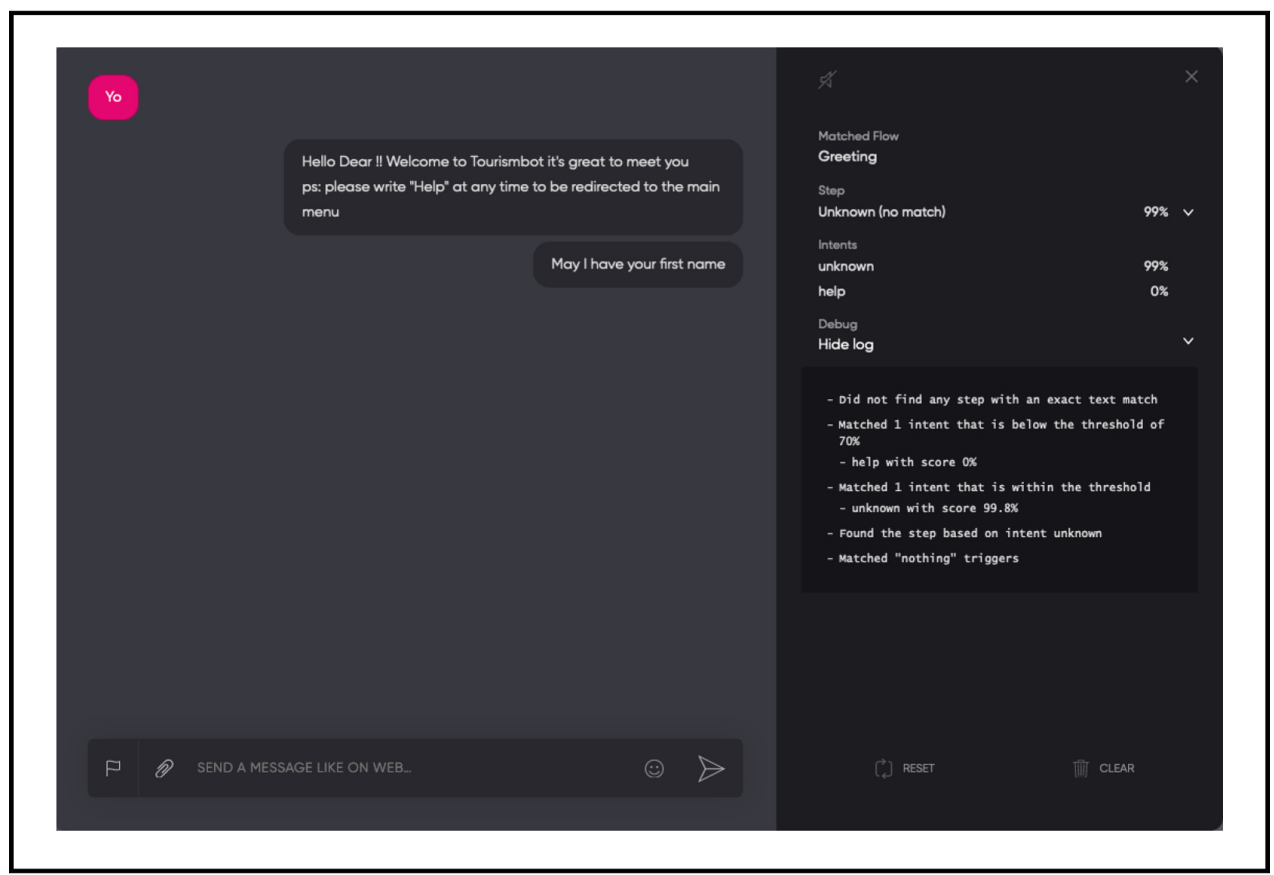

PAGE 16 JOURNAL OF TOURISM FUTURES $\mid$ VOL. m. NO. 2021 


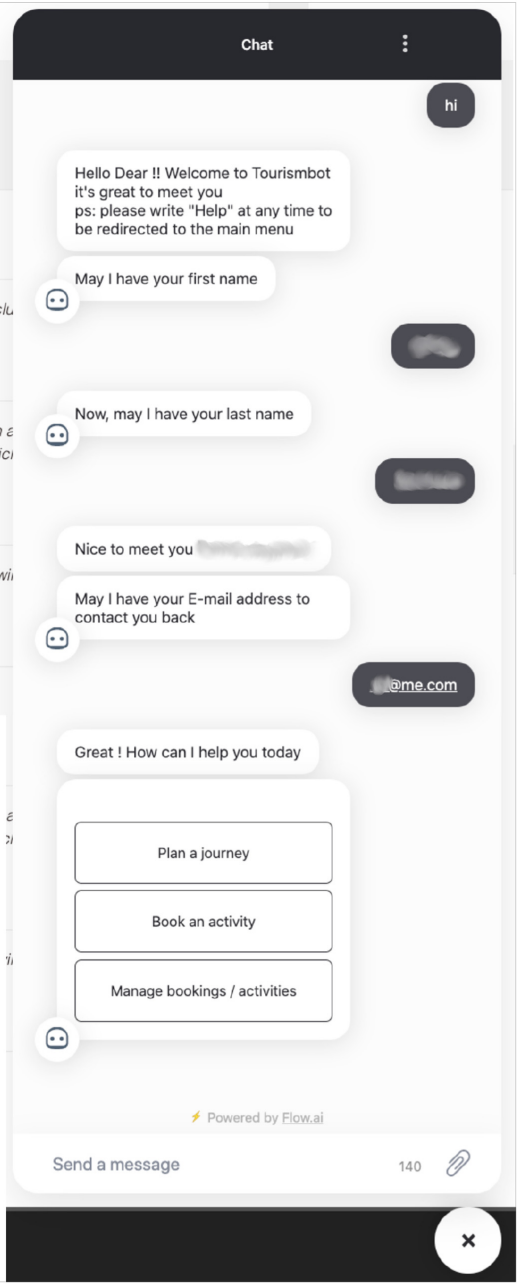

From greetings to main menu (a)

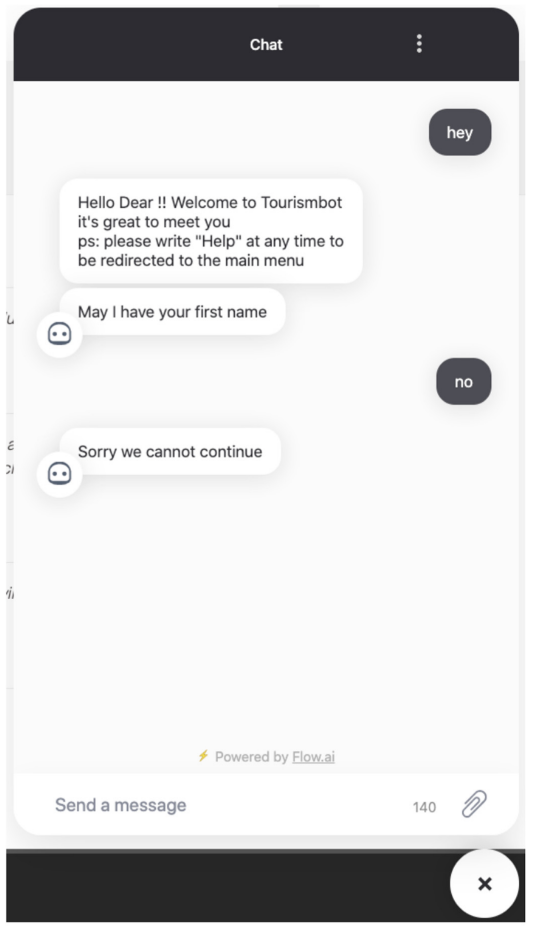

Interaction interrupted

(b)

4. Book an activity: Such a functionality can be performed both after booking a hotel (so in the same destination of such a venue, see Figure 9a) or independently from currently planned (proposing the destination slider as the first question, see Figure 8a). In turn, the user is required to select the activity (among the proposed ones, Figure 9b) day/period and the user's level in the chosen activity (Figure 9c). Such elements are used to cluster the tourists. If overlaps occur, the users are redirected to a thematic group chat (in this PoC, we used the WhatsApp platform, see Figure 10b).

5. Manage bookings: The user can delete and modify the booking performed in the current (cached) interaction.

The interactions characterizing the proposed PoC have been kept rather simplistic to allow the users (testers) to focus more on the functional possibilities unveiled by the realization of such a system rather than diverge their attention toward Human-Machine Interaction (HMI)-related topics (see Figure 11). 


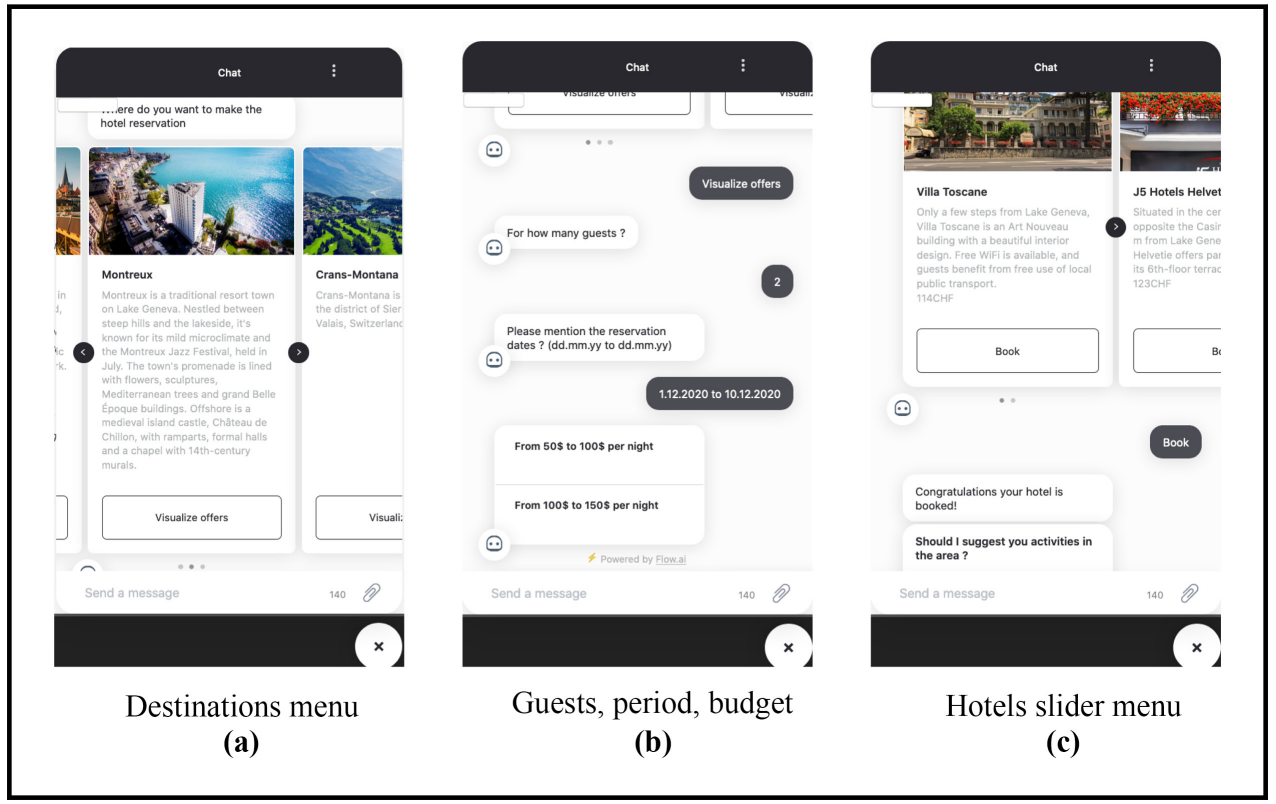

\section{Figure 9 Activities-related interactions}

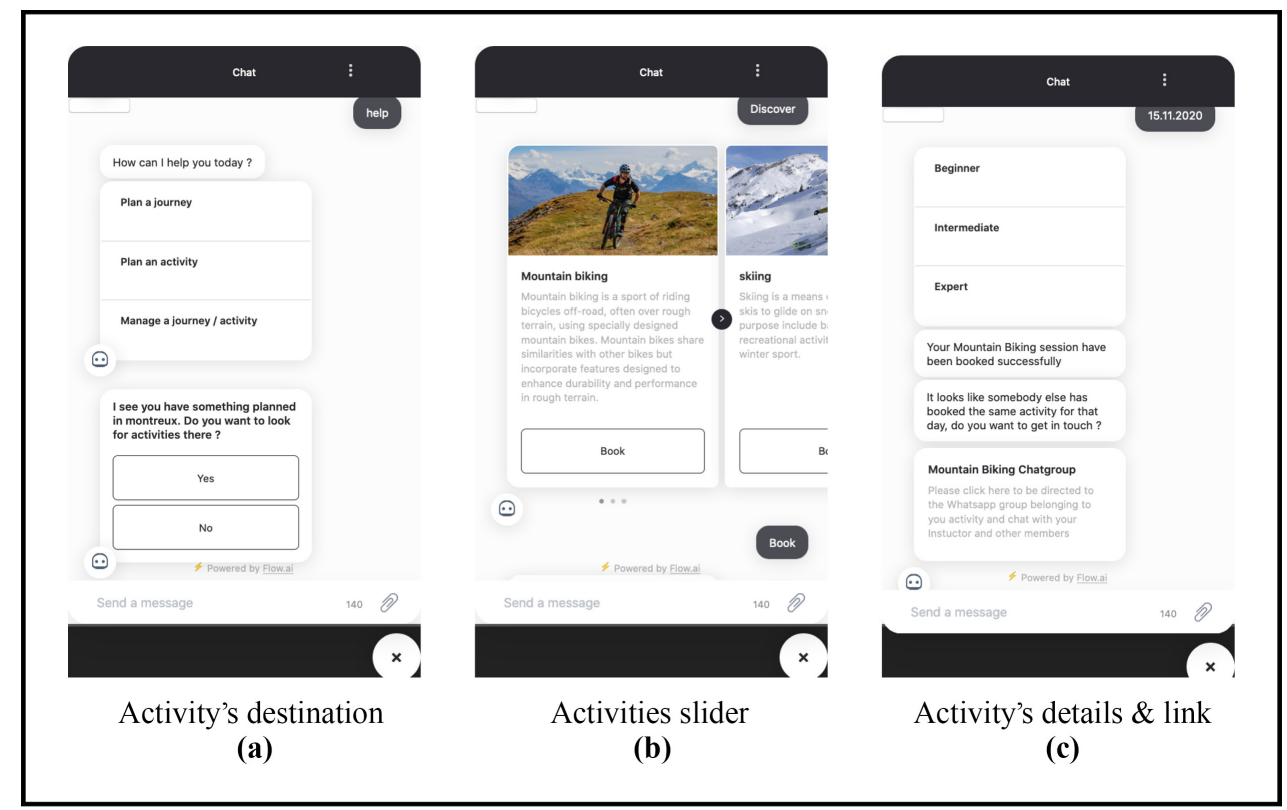

Figure 12 schematizes the PoC's workflow and the overall connection of the functionalities mentioned above.

To facilitate the testing, the PoC has been hosted on a dedicated website giving basic contextual information. In particular, the website has a responsive interface which allowed a fluid experience in multi-devices settings. 


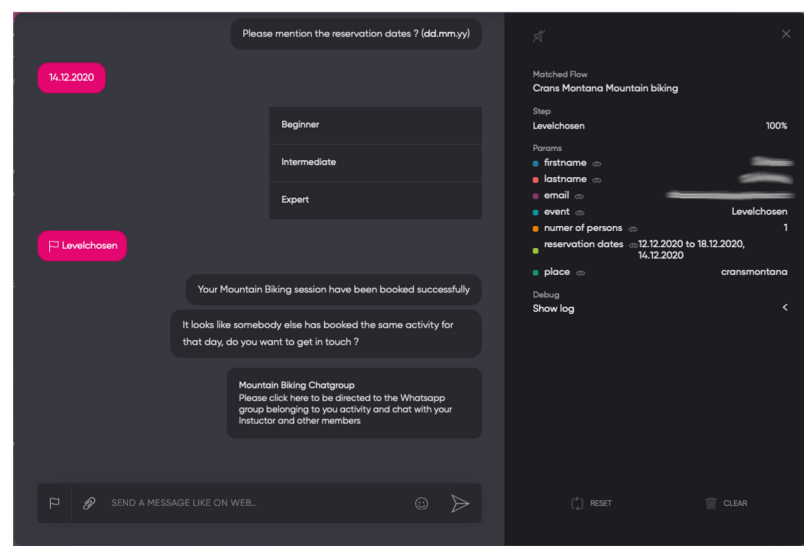

Flow.ai - Debug interface

(a)

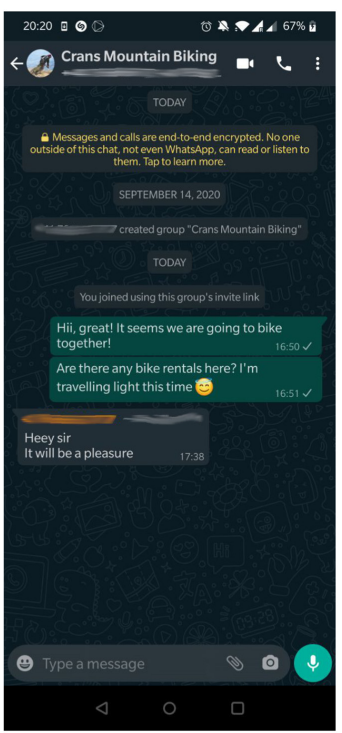

WhatsApp group

(b)

\section{Figure 11 PoC web and mobile interfaces}

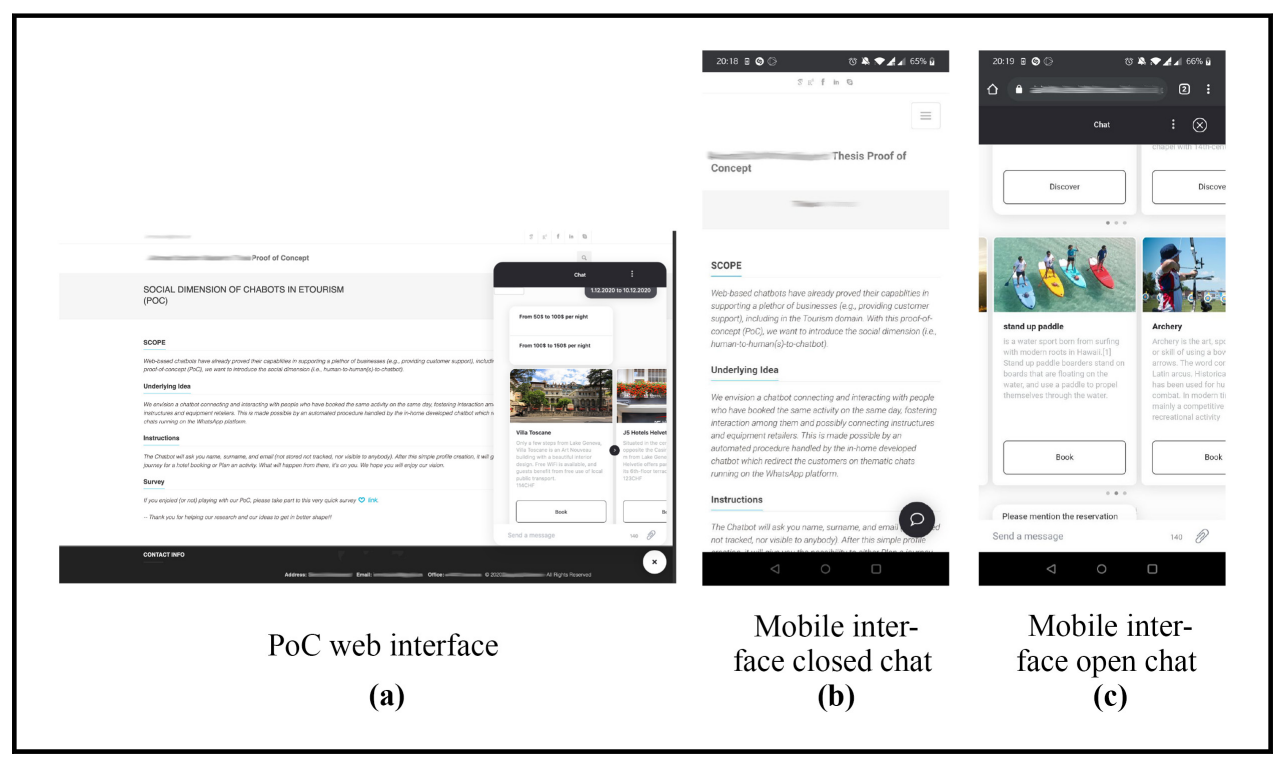

\subsection{PoC testing}

The testing of the PoC has lasted one month. However, the peak of the interactions has been registered in the first 10 days. Among the 18 people who tested the PoC, 7 were the experts who participated in the FG. Figure 13a shows the number of exchanged messages during the testing period. 


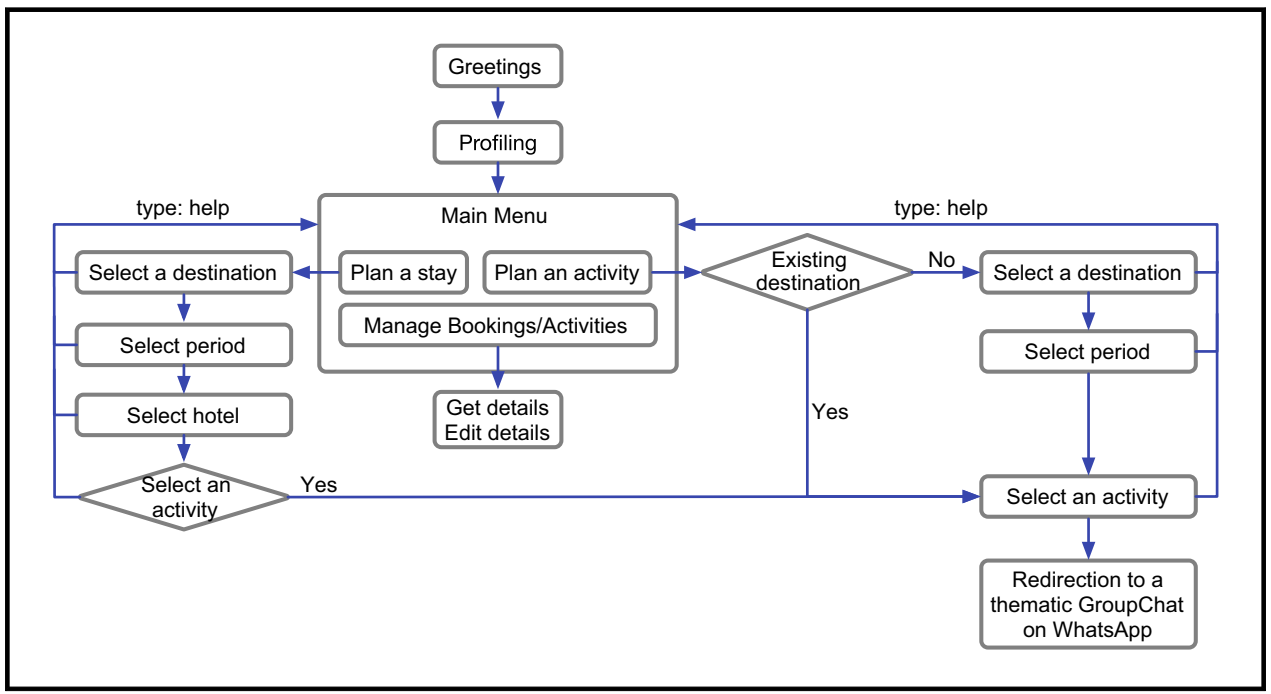

\section{Figure 13 PoC testing summary}

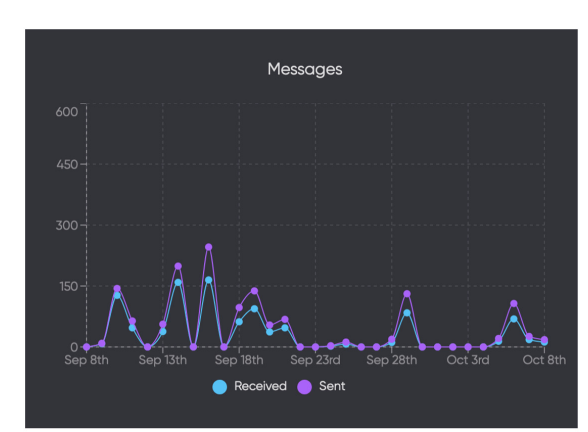

Messages exchanged over 1 month of testing

(a)

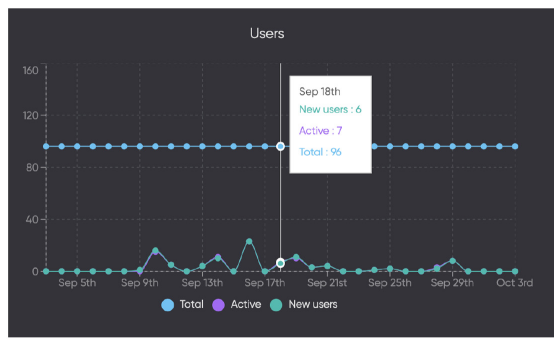

Number of users per day

(c)

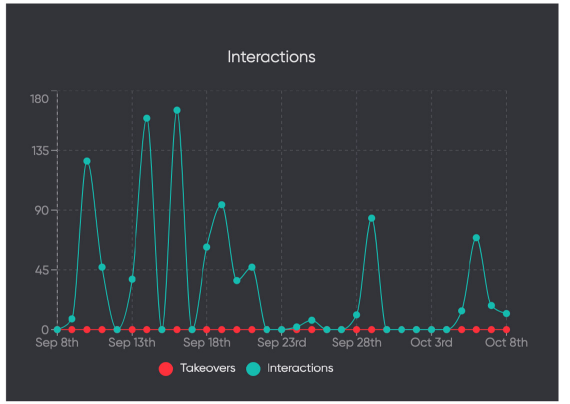

Number of sessions over 1 month of testing

(b)

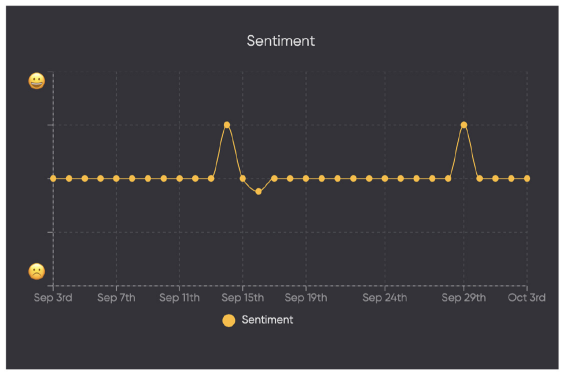

Messages' tone trend over 1 month

(d)

Among the 2800 exchanged messages, more than half have been sent by the bot to the user. This is due to the fact that during the design phase, the division of the messages has been thought to assume a more natural flow (i.e. sending two distinct messages instead of a longer one). According to the analytics provided by Flow.ai, a session is considered concluded after 15 min of inactivity between the $\mathrm{CB}$ and the user. The $\mathrm{CB}$ has recorded several, both sequential and 
contemporary, activations for a total of 1,022 sessions (see Figure 13b). Figure $13 \mathrm{c}$ shows that the system has recorded 96 different new activations (counted as different users). Being only 18 the unique real users, this means that some testers have restarted/re-initialized the CB to try the several interaction pathways and possible combinations. Overall, according to the sentiment analysis system provided by Flow.ai, the tones of the interactions have been mostly neutral with two remarkably positive and one minor negative exception (see Figure 13d).

\subsection{PoC survey}

In the web interface dedicated to the PoC, along with the contextual information, we have provided the link of the survey evaluating the user experience (see Figure 11a).

The survey consisted of the following eight questions (SVQs).

SVQ1: How did you find the underlying idea of the Proof-of-concept?

SVQ2: How do you see the social dimension in the tourist(s) - $\mathrm{CB}(\mathrm{s})$ interaction?

SVQ3: How would you rate your interaction with the CB?

SVQ4: For which activities would you employ this CB? (if any)

SVQ5: Any suggestions? (improvements or radical changes)

SVQ6: If available, would you use a CB embodying the presented concept to book Hotels/ Activities/etc.?

SVQ7: What did you like the most?

SVQ8: What did you dislike the most?

Besides six (out of eight) experts who participated in the FG, ten more experts and practitioners operating in the tourism and contingent sectors took part in the survey. Figure 14 summarizes the background of the participants.

Figure 15a shows how the participants have judged the PoC's idea quite positively. The interest confirmed after testing the $\mathrm{PoC}$ confirms the earlier positive responses manifested in the FG when only the idea has bad been introduced.

The idea of letting the CBs seamlessly aggregating tourists sharing interests and time-windows for the same activity(ies) as well as service or equipment providers have been largely appreciated (see

\section{Figure 14 Backgrounds of the survey's participants}

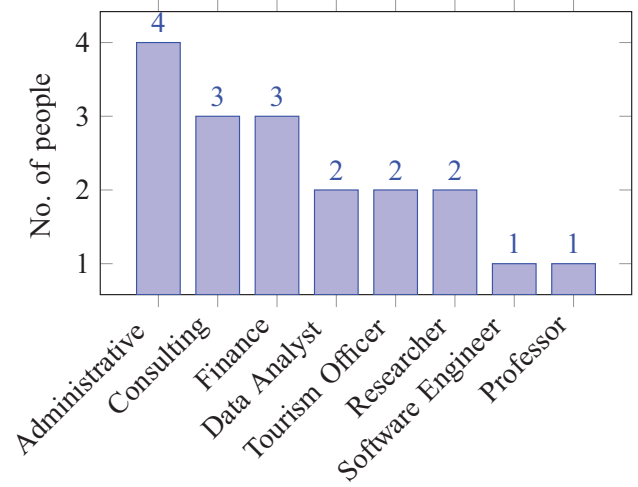

VOL. m. NO. m 2021 JOURNAL OF TOURISM FUTURES | PAGE 21 


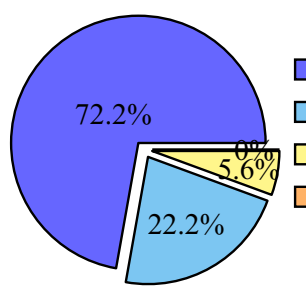

PoC's underlying idea

(a)
Very interesting

Interesting

Marginally interesting

Not interesting

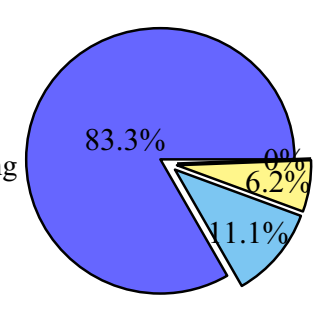

Social Dimension

(b)

Figure 15b). The PoC's underlying idea has received more approvals than the PoC itself (quite simplistic yet effective). Indeed, the ratios among the marks in both SVQ1 and SVQ2 of testers who have/have not participated in the FG are similar. While testers who found marginally interesting both the PoC (SVQ1) and the idea (SVQ2) remained of 5.6\%, the very interesting mark went up from $72.2 \%$ (SVQ1) to 83.3\% (SVQ2).

Concerning SVQ3, the interaction with the CB has been evaluated mostly satisfactory. In particular, the majority rated it 5/5 (44.4\%) and $4 / 5$ (44.4\%). One vote for both $3 / 5$ and $1 / 5(5.6 \%)$, and none for $2 / 5$ (see Figure 16). The appreciation of the interaction can also be suggested by the number of messages $(2,800)$ exchanged.

Table 2 collects the possible activities the testers envision for the employment of a similar chatbot (SVQ4). Overall, when such a CB will be finalized, the testers desire it in charge of the micromanaging of activities spanning from those offered by a sole hotel/facility to those available in a whole district/territory.

The testers have been asked about possible suggestions to improve or radically change the CB tested in the PoC. Table 3 shows the responses collected. Among these, we can highlight the need for personalization and customization, which is perceived as key for user engagement and for satisfying specific customer needs. Moreover, the use of voice interactions is of particular interest, as it can boost the use of hand-free instructions. Other comments refer to different communication strategies, as well as privacy concerns regarding data sharing or payment details.

As mentioned above, we have opted for deploying the PoC on a dedicated webpage to facilitate its understanding and use. Nevertheless, the CB could be deployed in existing, plugged as a thirdparty component, or having a dedicated application. SVQ6 investigates the preferences expressed by testers (see Figure 17).

\section{Figure 16 Backgrounds of the survey's participants}

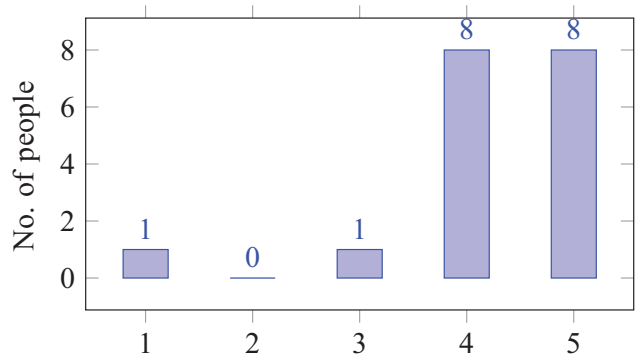

PAGE 22 |JOURNAL OF TOURISM FUTURES $\mid$ VOL. $\mathbf{m}$ NO. $\mathbf{m} 2021$ 


\section{Table 2 Proposed future applications for the proposed PoC}

\# Suggestion

Services and activities reservation and management

Booking hotels and leisure activities

Any activity that requires coaching

Managing common/group tasks beyond tourism (e.g. banks, embassies and government institutions)

All kind of activities that can be offered by a hotel

To get in contact with people that are interested in the same activities as me

Booking and managing independently accommodation and activities

Customers welcoming

Simplifying back- and front-office interconnection

10 All kind of activities

All the activities that might require aggregations (e.g. museum and swimming pool)

Conciergerie

Sport but most importantly boost cultural activities and knowledge sharing

Personal tutor for tourists and more

Crowd management for outdoor events

Multi-facility vacations

City or natural reserves (walking) explorers

Promoting small local businesses

\section{Table 3 Proposed future applications for the proposed PoC}

\begin{tabular}{|c|c|}
\hline & uggestion \\
\hline $1-4$ & No \\
\hline 5 & More suggestions \\
\hline 6 & Increase the granularity and complete it (l'd love to try it when finalized) \\
\hline 7 & $\begin{array}{l}\text { Additional capabilities (e.g. created favorites activities/people, and price comparison between } \\
\text { customizable destinations) }\end{array}$ \\
\hline 8 & Don’t ask the name as first thing, it might be intimidating \\
\hline 9 & Enable vocal interaction \\
\hline 10 & $\begin{array}{l}\text { More ways of connecting tourists no just via existing app such as WhatsApp which are not fully } \\
\text { trusted! }\end{array}$ \\
\hline 11 & Add widget such as calendar to pick the time window \\
\hline 12 & $\begin{array}{l}\text { Include the responsible of the activity or event in the WhatsApp group (to answer participants' } \\
\text { questions and interact with them) }\end{array}$ \\
\hline 13 & Chatbot needs a voice \\
\hline 14 & Customizable confidence level, the chatbot should not call me dear by default \\
\hline 15 & $\begin{array}{l}\text { I would not trust to give my credit card details in a chat, booking and payment needs to be finalized } \\
\text { elsewhere }\end{array}$ \\
\hline 16 & Simplified data selection, clear organization of bookings and activities \\
\hline 17 & Maybe connected to a web interface \\
\hline 18 & $\begin{array}{l}\text { Customizable level of information sharing. I would not trust to allow strangers to have my contact } \\
\text { details through a group chat }\end{array}$ \\
\hline
\end{tabular}

Finally, we asked, in general, the tester opinion about the overall experience (from the context to the possible applications), asking to indicate what has been appreciated the most (see Table 4) and what has been disliked the most (see Table 5), if anything.

\section{Discussion and conclusions}

The study conducted on the SLR, FG and PoC highlighted several crucial aspects for researchers and practitioners in the Tourism Domain. In particular, the limited knowledge shown about CBs (either about what they are or what they can do) is the most limiting and concerning aspect. In turn, the lack of knowledge about data protection regulations is a relevant setback that raises limits to the potential (and ethics) of the several data-analysis mechanisms running in the back-end of 


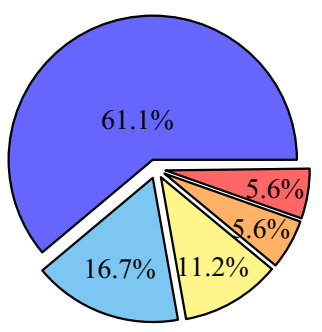

Yes - on website(s)

Yes - on dedicated App(s)

$\square$ Yes - combined in new \& existing App(s)

$\square$ Yes - must be multi-platform

Nowhere - I prefer old ways

\section{Table 4 Most liked aspects}

\# Suggestion

1 The interaction

2 The CB intelligence and capabilities

3 Getting fast, specific and clear answers

The interactivity and potential connection with others who have the same itinerary

The interactivity and the design

The bot responds quite fast and has a nice design. The structure is logical

7 It is a very interesting idea of creating a chatbot in tourism sphere. The structure of the chatbot is very easy to use, where it is possible to book hotels in different price categories. Additionally with this chatbot, you can plan your activities, and once you have booked your activities, the chatbot offers you to join Chatgroup, which is such a good idea and possibility to meet new people for the same dates of vacation with the same interests in activities in advance

8 The potential this chatbot expresses

9 The fact that the bot optimize the customer journey of the tourist directly from the supply side

10 Easy of use and recommendations

11 Interface

12 The pictures and the user-friendliness

13 Exact and constant mood

14 The speed of answer, the various suggestions

15 The very interesting concept

16 The smooth flow and the selectors

17 Generating new business models

18 Concrete possibilities for multi-party investments

\section{Table 5 Most disliked aspects}

\# Suggestion

$1 \quad$ Nothing in particular, I have really liked the proof-of-concept which has been proposed quite rapidly after the focus group

2 The final version needs to be extremely more personalized

$3 \quad$ No flaws spotted at the moment

$4 \quad$ Nothing worth to be mentioned at this stage

$5 \quad$ I'm super curious, why is not ready yet?:)

6 Such reflection should put more humans at the center and not the technology

$7 \quad$ Nothing

8 The lack of condition control in the answer (format email and date) but it's a POC;-)

9 Robotic tone

10 Privacy concerns

11 The descriptions of the hotels were too long so annoying to scroll

12-18 N/A 
current CBs. Moving analysis from the academic contributions to the industrial FG, several aspects have been confirmed. The most relevant for our investigations is the incapability of foreseeing the social dimension applied to CBs, tourists and service providers. The FG also confirms the results of the SLR regarding the need for further personalization of chatbot interactions. This includes not only the customization of how the chatbot behaves but also how it adapts its strategy and anticipates user's actions and preferences, thanks to advanced profiling and data-driven analysis capabilities.

In this regard, several challenges arise from the fact that chatbots are moving from simple automatons to advanced Al-powered autonomous agents. One key aspect to investigate in the future is related to the correct assessment of chatbot knowledge. Given that personalized features and user patterns feed the beliefs and knowledge of the bot, it becomes crucial to study techniques for evaluating what the chatbot learns. This also includes exploring misinterpretation issues, e.g. when it is the human who introduces errors at a certain point of the interaction. Transparent and explainable knowledge is required in order to address this challenge, which also has an impact on the potential trust in the chatbot interactions.

Finally, as it is discussed previously, the POC described in this paper shows the potential of social interactions through conversational agents, exploiting synergies among different tourism service providers. Beyond technological advancements, this concept also includes modeling dynamics, activities and tourist journeys, reconciling multiple and diverse behavior patterns. The results of our survey show that stakeholders need to be involved and participate more closely in the analysis of the potential development of such services in order to understand if the market is even ready for it and to what specific customer needs it responds to. This entails exploring the intention of customers and users with respect to Al chatbot technologies, and their corresponding adoption models (Pillai and Sivathanu, 2020). Moreover, it is important to understand how it fits the overall business model, considering the involvement of tourism consortia and the markets it may generate. Finally, particular attention has to be given to the potential impact of the "chatbots-social leverage" in tourism. Deploying the dynamics proposed in the POC can improve the quality of existing services - hence, the user satisfaction - and, most importantly, radically reshape several decision processes and policies. Therefore, as ongoing and future work, we plan to finalize and deploy a more complex prototype in small Swiss touristic venues. It will widen our understanding of the envisioned potential, identifying which touristic segments, tourist typologies, destinations and activities can profit the most and how they can be inter-correlated.

\section{References}

Amalia, A. and Suprayogi, M. (2019), "Engaging millennials on using chatbot messenger for eco-tourism", Third International Conference on Sustainable Innovation 2019-Humanity, Education and Social Sciences (ICOSIHESS 2019), Atlantis Press.

Arteaga, D., Arenas, J., Paz, F., Tupia, M. and Bruzza, M. (2019), "Design of information system architecture for the recommendation of tourist sites in the city of manta, ecuador through a chatbot", 2019 14th Iberian Conference on Information Systems and Technologies (CISTI), IEEE, pp. 1-6.

Bozic, J., Tazl, O.A. and Wotawa, F. (2019), "Chatbot testing using Al planning", 2019 IEEE International Conference on Artificial Intelligence Testing, pp. 37-44.

Buhalis, D. and Yen, E.C.S. (2020), "Exploring the use of chatbots in hotels: technology providers' perspective", Information and Communication Technologies in Tourism 2020, Springer, pp. 231-242.

Calvaresi, D., Cesarini, D., Sernani, P., Marinoni, M., Dragoni, A. and Sturm, A. (2016), "Exploring the ambient assisted living domain: a systematic review", Journal of Ambient Intelligence and Humanized Computing, Vol. 8 No. 2, pp. 1-19.

Calvaresi, D., Appoggetti, K., Lustrissimini, L., Marinoni, M., Sernani, P., Dragoni, A.F. and Schumacher, M. (2018), 'Multi-agent systems' negotiation protocols for cyber-physical systems: results from a systematic literature review", Proceedings of ICAART.

Calvaresi, D., Calbimonte, J.-P., Dubosson, F., Najjar, A. and Schumacher, M. (2019), "Social network chatbots for smoking cessation: agent and multi-agent frameworks", 2019 IEEE/WIC/ACM International Conference on Web Intelligence (WI), IEEE, pp. 286-292. 
Calvaresi, D., Ibrahim, A., Calbimonte, J.-P., Schegg, R. and Schumacher, M. (2021), "The evolution of chatbots in tourism: a systematic literature review", Proceeding of the 28th annual international eTourism conference ENTER2021, Springer (to appear).

Ceccarini, C. and Prandi, C. (2019), "Tourism for all: a mobile application to assist visually impaired users in enjoying tourist services", 2019 16th IEEE Annual Consumer Communications and Networking Conference (CCNC), IEEE, pp. 1-6.

Chaves, A.P. (2020), "Should my chatbot be register-specific? designing appropriate utterances for tourism", Extended Abstracts of the 2020 CHI Conference on Human Factors in Computing Systems, pp. 1-11.

Chrysovelidis, G. (2020), "Designing a chatbot for tourism”, Master's thesis, International Hellenic University of Thessaloniki.

Clarizia, F., Colace, F., De Santo, M., Lombardi, M., Pascale, F. and Santaniello, D. (2019), "A context-aware chatbot for tourist destinations", 2019 15th International Conference on Signal-Image Technology and Internet-Based Systems (SITIS), IEEE, pp. 348-354.

cvent (2019), Hotel Chatbots: Your New Best Friends for Creating a Great Customer Experience.

Dyrkolbotn, S., Pedersen, T. and Slavkovik, M. (2018), "On the distinction between implicit and explicit ethical agency", Proceedings of the 2018 AAAl/ACM Conference on Al, Ethics, and Society, pp. 74-80.

Flow.ai (2020), "Intelligent automation using bots", available at: https://flow.ai/.

Hadnagy, C. (2010), Social Engineering: The Art of Human Hacking, Wiley \& Sons.

Haque, M.M. (2019), "Slackbot design and development", Bachelor's Thesis, Turku University of Applied Sciences.

Hashim, N.L. and Isse, A.J. (2019), "Usability evaluation metrics of tourism mobile applications", Journal of Software Engineering and Applications, Vol. 12 No. 7, pp. 267-277.

Hosseini, S. (2020), “Using a chatbot to increase tourists' engagement”, Bachelor's thesis, LAB University of Applied Sciences.

Hu, T., Xu, A., Liu, Z., You, Q., Guo, Y., Sinha, V., Luo, J. and Akkiraju, R. (2018), “Touch your heart: a toneaware chatbot for customer care on social media", Proceedings of the $\mathrm{CHI}$ Conference on Human Factors in Computing Systems, pp. 1-12.

Ivanov, S.H. (2019), "Ultimate transformation: how will automation technologies disrupt the travel, tourism and hospitality industries?", Zeitschrift für Tourismuswissenschaft, Vol. 11 No. 1, pp. 25-43.

Ivanov, S.H. (2020), "The first chatbot of a tourism/hospitality journal: editor's impressions", Hospitality Journal: Editor's Impressions (March 1, 2020), European Journal of Tourism Research, Vol. 24, p. 2401.

Ivanov, S.H. and Webster, C. (2017), "Adoption of robots, artificial intelligence and service automation by travel, tourism and hospitality companies-a cost-benefit analysis", Artificial Intelligence and Service Automation by Travel, Tourism and Hospitality Companies-A Cost-Benefit Analysis.

Jindal, G., Upadhyay, D. and Jha, A. (2020), “Whatsapp chatbot”, Technical report, EasyChair.

Kasinathan, V., Abd Wahab, M.H., Idrus, S.Z.S., Mustapha, A. and Yuen, K.Z. (2020), "Aira chatbot for travel: case study of Airasia", Journal of Physics: Conference Series, Vol. 1529, No. 2, p. 022101.

Kitchenham, B., Pearl Brereton, O., Budgen, D., Turner, M., Bailey, J. and Linkman, S. (2009), "Systematic literature reviews in software engineering - a systematic literature review", Information and Software Technology, Vol. 51 No. 1, pp. 7-15.

Kitchenham, B., Brereton, P., Turner, M., Niazi, M., Linkman, S., Pretorius, R. and Budgen, D. (2010), "Refining the systematic literature review process-two participant-observer case studies", Empirical Software Engineering, Vol. 15 No. 6, pp. 618-653.

Lombardi, M., Pascale, F. and Santaniello, D. (2019), "An application for cultural heritage using a chatbot”, 2019 2nd International Conference on Computer Applications and Information Security (ICCAIS), IEEE, pp. 1-5.

Lukanova, G. and llieva, G. (2019), "Robots, artificial intelligence, and service automation in hotels", Robots, Artificial Intelligence, and Service Automation in Travel, Tourism and Hospitality, Emerald Publishing, pp. 157-183.

Machine, T. (2018), Facebook Messenger Passes 300,000 Bots. 
Melián-González, S., Gutiérrez-Taño, D. and Bulchand-Gidumal, J. (2019), "Predicting the intentions to use chatbots for travel and tourism", Current Issues in Tourism, Vol. 24 No. 2, pp. 192-210.

Morze, N., Buinytska, O. and Varchenko-Trotsenko, L. (2017), "Use of bot-technologies for educational communication at the university", Technical report, Studio NOA for University of Silesia in Katowice.

phocuswire (2018), How Cutting-Edge Hotels Use Artificial Intelligence for a Great Guest Experience.

Pillai, R. and Sivathanu, B. (2020), "Adoption of Al-based chatbots for hospitality and tourism”, International Journal of Contemporary Hospitality Management, Vol. 32 No. 10, pp. 3199-3226.

Popesku, J. and Zlatanov, S. (2019), "Current applications of artificial intelligence in tourism and hospitality", Sinteza 2019-International Scientific Conference on Information Technology and Data Related Research, Singidunum University, pp. 84-90.

Quicktext (2019a), How Big Hospitality Brands Are Leveraging Al Today.

Quicktext (2019b), Tech Japan's Henn-Na Hotel Fires Half its Robot Workforce.

Ruf, B., Sammarco, M., Aigrain, J. and Detyniecki, M. (2020), "Pharmabroad: a companion chatbot for identifying pharmaceutical products when traveling abroad", Information and Communication Technologies in Tourism 2020, Springer, pp. 218-228.

Sano, A.V.D., Imanuel, T.D., Calista, M.I., Nindito, H. and Condrobimo, A.R. (2018), "The application of agnes algorithm to optimize knowledge base for tourism chatbot", 2018 International Conference on Information Management and Technology (ICIMTech), IEEE, pp. 65-68.

Ukpabi, D.C., Aslam, B. and Karjaluoto, H. (2019), Chatbot Adoption in Tourism Services: A Conceptual Exploration. Robots, Artificial Intelligence, and Service Automation in Travel, Tourism and Hospitality, Emerald Publishing, pp. 105-121.

Weizenbaum, J. (1966), "Eliza-a computer program for the study of natural language communication between man and machine", Communications of the ACM, Vol. 9 No. 1, pp. 36-45.

\section{Corresponding author}

Davide Calvaresi can be contacted at: davide.calvaresi@hevs.ch

For instructions on how to order reprints of this article, please visit our website: www.emeraldgrouppublishing.com/licensing/reprints.htm Or contact us for further details: permissions@emeraldinsight.com 